\title{
Modeling and Experiment of a V-Shaped Piezoelectric Energy Harvester
}

\author{
Yue Zhao, ${ }^{1,2}$ Yi Qin $\left(\mathbb{D},{ }^{1,2}\right.$ Lei Guo, ${ }^{1,2}$ and Baoping Tang ${ }^{1,2}$ \\ ${ }^{1}$ State Key Laboratory of Mechanical Transmission, Chongqing University, Chongqing 400044, China \\ ${ }^{2}$ College of Mechanical Engineering, Chongqing University, Chongqing 400044, China \\ Correspondence should be addressed to Yi Qin; qy_808@aliyun.com
}

Received 31 July 2017; Accepted 25 September 2017; Published 27 May 2018

Academic Editor: Paul Cahill

Copyright (c) 2018 Yue Zhao et al. This is an open access article distributed under the Creative Commons Attribution License, which permits unrestricted use, distribution, and reproduction in any medium, provided the original work is properly cited.

\begin{abstract}
Vibration-based energy harvesting technology is the most promising method to solve the problems of self-powered wireless sensor nodes, but most of the vibration-based energy harvesters have a rather narrow operation bandwidth and the operation frequency band is not convenient to adjust when the ambient frequency changes. Since the ambient vibration may be broadband and changeable, a novel V-shaped vibration energy harvester based on the conventional piezoelectric bimorph cantilevered structure is proposed, which successfully improves the energy harvesting efficiency and provides a way to adjust the operation frequency band of the energy harvester conveniently. The electromechanical coupling equations are established by using Euler-Bernoulli equation and piezoelectric equation, and then the coupled circuit equation is derived based on the series connected piezoelectric cantilevers and Kirchhoff's laws. With the above equations, the output performances of V-shaped structure under different structural parameters and load resistances are simulated and discussed. Finally, by changing the angle $\theta$ between two piezoelectric bimorph beams and the load resistance, various comprehensive experiments are carried out to test the performance of this V-shaped energy harvester under the same excitation. The experimental results show that the $\mathrm{V}$-shaped energy harvester can not only improve the frequency response characteristic and the output performance of the electrical energy, but also conveniently tune the operation bandwidth; thus it has great application potential in actual structure health monitoring under variable working condition.
\end{abstract}

\section{Introduction}

Recently, wireless sensor networks (WSNs) have received much attention. WSNs technology has been recognized as an effective technique for the various industrial fields [1], such as environmental monitoring [2], fire detection [3], body area networks [4], and fault diagnosis [5]. But the limited energy associated with WSNs is a great obstacle of WSN technologies. Environmental energy harvesting is the most probable way to overcome this major bottleneck. For example, vibration energy, solar energy, wind energy, thermal energy, and other green energy [6] can be converted into electrical energy which can augment an existing battery supply or replace it entirely given certain conditions.

Since vibration widely exists in mechanical equipment, bridge, and human body, the commonly used energy harvesting is based on vibration. The possible vibration-to-electric energy conversion mechanisms include piezoelectric, electromagnetic, and electrostatic transductions, which provide a high power density [7]. Among these three alternatives for vibration-to-electric energy conversion, piezoelectric transduction has received the most attention. Rocha et al. [8] proposed a system integrated in footwear to harvest energy from vibration when people walk. In [9], a piezoelectric energy harvester is developed to harvest energy from the acceleration of tires. P Gambier et al. [10] designed hybrid low-power generator systems with thin-film batteries and storage system using piezoelectric, solar, and thermal sources of energy in order to improve both structural multifunctionality and system-level robustness in energy harvesting.

The most commonly used piezoelectric energy harvester configuration is a cantilever with piezoceramic layers located on a vibrating host structure for electrical power generation from bending vibrations under resonance excitation [12-15]. Various piezoelectric transductions are designed to harvest energy in a single direction, whereas they have better energy conversion efficiency only when the excitation frequencies of the vibration sources closely match the resonance frequencies 
of the devices [16-19]. Unfortunately, when the ambient vibration sources are multidirectional and have a wide spectrum, these piezoelectric harvesters [20] will probably lose their efficacy.

To overcome the above problems, various vibration energy harvesters which are suitable for multidirectional vibrations or wide-band vibrations have been explored. For example, Leadenham et al. [21] presented an M-shaped nonlinear bent beam with clamped end conditions and investigated for bandwidth enhancement under base excitation. Harne et al. [22] investigated the versatility of internal resonance-based behaviors for energy harvesting analytically, numerically, and experimentally by exploiting nonlinear, multimodal motions in an L-shaped energy harvester platform. Danzi $\mathrm{F}$ et al. $[23,24]$ investigated to use a topology optimization to design planar resonators and derived a reduced order model to further investigate the dependence of the beams' orientation on the frequencies ratio. These results show an infinite number of topologies that can have integer ratio modal frequencies and in some cases harvest more power than a nominal L-shaped harvester, operating in the linear regime. Kim et al. [25] and Andò et al. [26] designed two-dimensional piezoelectric energy harvester, respectively, which is sensitive to the vibration of two directions. Su et al. [27] studied the three-directional harvester by using spring and magnet. The method to design the multidirectional piezoelectric energy harvester is to optimize the structure so that the harvester could work effectively by inertia when excited at some direction. In order to broaden the effective operation frequency band of the harvester, a multiresonant piezoelectric beam was used by attaching multiple cantilever beams of natural frequencies close to the exciting frequency $[28,29]$. Yang et al. [30] proposed a power harvester that consisted of two piezoelectric beams connected with elastic springs, and it could also improve the bandwidth. Another approach is to provide a resonance tuning mechanism. Hu et al. [31] studied the technique to adjust the flexural mode of a piezoelectric bimorph through axial preloads, which is useful for scavenging energy from ambient vibration with varying frequency spectra. Challa et al. [32] presented a vibration energy harvesting device with autonomous resonance frequency tenability by utilizing a magnetic stiffness technique. In addition, there are other methods to broaden the operating frequency bandwidth of the energy harvester, such as monostable and bistable mechanisms [33-36] and piecewise linear mechanisms [37]. However, their tuning mechanisms make the structure of these harvesters more complex, which may hamper their application in engineering. What is more, the monostable and bistable mechanisms will have high conversion efficiency only when the excitation amplitude is large. In many cases, the amplitude of the environmental vibration is small, and it is difficult to sustain the high output of the harvester. From the above, it can be known that most of the energy harvesters seldom pay attention to multidirection and wide operation bandwidth simultaneously.

As an alternative to conventional multiresonant piezoelectric beams, Erturk A et al. [38] proposed an L-shaped beam-mass structure as a new piezoelectric energy harvester configuration, which can broaden the operation bandwidth and overcome the shortcomings of the single frequency excitation. However, in their study, the angle of the Lshaped harvester is fixed; thus it is difficult to be applicable to the variable working condition. Su et al. [11] further provided detailed modeling of the $\mathrm{V}$-shaped structure on the basis of Euler-Bernoulli beam theory and compared the voltage and power frequency responses under various angles between the two beams. The V-shaped structure consisted of aluminum substructure and unimorph piezoelectric layer, which can effectively broaden the bandwidth. Nevertheless, the generated voltage and power are rather small, which is difficult to meet the requirements of the actual application.

Based on the above considerations, a new V-shaped piezoelectric low frequency vibration energy harvester which can arbitrarily tune the angle between the two piezoelectric bimorph beams is explored in this study. The electromechanical coupling model of the proposed harvester is built up, and then the simulation and experiments are performed under different structural parameters and load resistances. The frequency of the peak voltage is the natural frequency (resonant frequency). When the designed harvester is excited at natural frequency, the most power output can be achieved by resistance matching. Like the previous $\mathrm{L}$-shaped or $\mathrm{V}$ shaped piezoelectric unimorph harvester, the proposed Vshaped harvester also can be used as a broader band energy harvesting system, whereas the comparative results show that the V-shaped harvester can not only improve the output performance of the electrical energy, but also enhance the frequency response characteristic of the operation frequency band in some case. In addition, the proposed V-shaped piezoelectric bimorph harvester can be conveniently tuned by the use of a proper connector; thus when the ambient exciting frequency changes, we can simply tune the angle between beam 1 and beam 2 so as to change the natural frequency and the operation bandwidth. It then follows that the proposed Vshaped harvester has great application potential and value in the health monitoring of complicated mechanical equipment and bridge.

This paper is organized as follows. In Section 2, an electromechanical coupling model is established by using the Euler-Bernoulli equation and piezoelectric equation. In Section 3, numerical simulations are carried out to test the performance of the V-shaped harvester. In Section 4, several experiments are performed under different intersection angles and load resistances to further validate the performance of the V-shaped harvester. Finally, the conclusions are presented in Section 5.

\section{V-Shaped Piezoelectric Energy Harvester Configuration and Its Mathematical Model}

2.1. Configuration of V-Shaped Harvester. Cantilever structure has been widely applied to vibration-to-electric energy transducer. Although the conventional single piezoelectric cantilevered structure can generate a large output voltage when it is excited at its resonance frequency, the output voltage of the converter would decrease sharply when the 


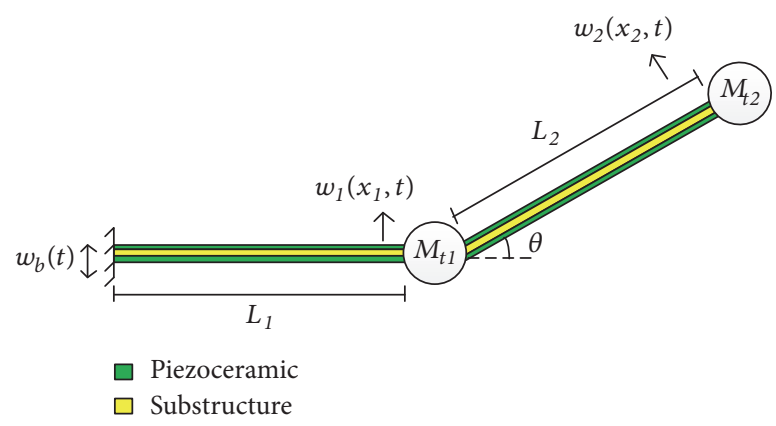

FIGURE 1: Schematic of the V-shaped piezoelectric energy harvester.

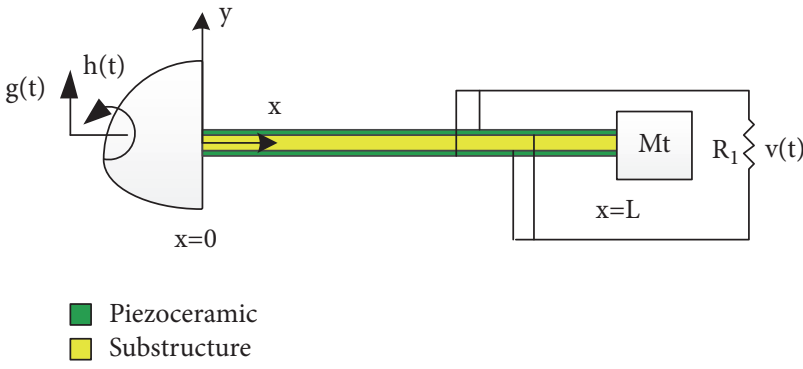

(a)

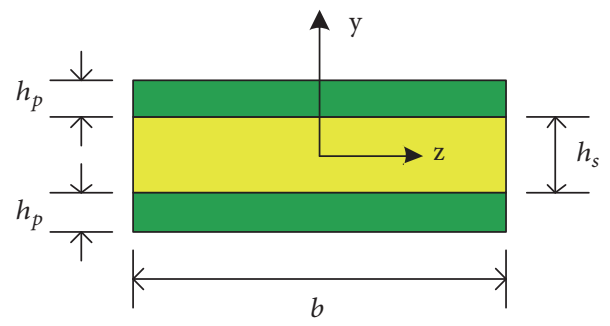

(b)

FIGURE 2: Schematic of a bimorph cantilever configuration with a tip mass: (a) parallel connection of piezoceramic layers and (b) crosssectional view of a bimorph cantilever.

excitation frequency is not consistent with its natural frequency. Furthermore, the conventional single piezoelectric harvester generates high power just within a narrow frequency range and is not convenient to adjust when the ambient frequency changes. In order to solve these problems, a V-shaped piezoelectric bimorph energy harvester is proposed in this study. Figure 2 illustrates the schematic of the V-shaped piezoelectric energy harvester. Obviously, this harvester consists of two cantilevered piezoelectric beams whose intersection angle is defined as $\theta$; and $w_{b}(t)$ is the ambient excitation. The length of beam 1 is $L_{1}$, and the length of beam 2 is $L_{2} ; M_{t 1}$ and $M_{t 2}$ are the tip masses of beam 1 and beam 2 , respectively. $w_{1}\left(x_{1}, t\right)$ is the transverse vibration response of beam 1 and $w_{2}\left(x_{2}, t\right)$ is the transverse vibration response of beam 2 . Both beam 1 and beam 2 are cantilevered piezoelectric bimorph beams, which are shown in Figure 1. In this figure, the green region represents the piezoelectric layer, and the yellow region represents the metal substrate.

2.2. Mechanical Coupling Equation. As shown in Figure 2, a bimorph piezoelectric cantilever with a tip mass is presented and the piezoelectric layers are connected in parallel. Based on the hypothesis of Euler-Bernoulli beam, the beam presented here can be recognized as a homogeneous composite beam and satisfies the linear deformation and small vibration conditions. So the influence of shear deformation and moment of inertia can be ignored. The piezoelectric bending structure is generally a thin-walled beam, which makes this assumption reasonable. In addition, the mechanical loss consists of the internal damping (strain) and the external damping (viscous air). Assuming that the piezoelectric layer and the substructure are integrated closely and the electrode layer covering the piezoelectric layer is very thin, so the influence of the electrode layer can be ignored. In order to ensure the integrity of the mathematical model deduction, the procedure details can be acquired in [39].

For a homogeneous composite beam, the partial differential equation in the transverse direction can be written as [40]

$$
\begin{aligned}
& \frac{\partial^{2} M(x, t)}{\partial x^{2}}+c_{s} I \frac{\partial^{5} w(x, t)}{\partial x^{4} \partial t}+c_{a} \frac{\partial w(x, t)}{\partial t}+m \frac{\partial^{2} w(x, t)}{\partial t^{2}} \\
& =f(x, t)
\end{aligned}
$$

where $M(x, t)$ is the moment of the beam at position $\mathrm{x}$ and time $t, c_{s} I$ is the equivalent damping term in the cross-section of the beam due to the strain rate damping, $c_{a}$ is the viscous air damping coefficient, $m$ is the mass of the beam per unit length, $w(x, t)$ is the absolute transverse motion of the beam at any position $x$ and time $t, f(x, t)$ is the external force at any position $x$ and time $t$.

The absolute transverse motion of the beam at any position $x$ and time $t$, and $w(x, t)$, can be written as

$$
w(x, t)=w_{b}(x, t)+w_{\text {rel }}(x, t)
$$

where $w_{b}(x, t)$ is the displacement of the base and $w_{r e l}(x, t)$ is the transverse deflection of the beam relative to its base.

As shown in Figure 2, the motion of the base $w_{b}(x, t)$ can be written as

$$
w_{b}(x, t)=g(t)+x h(t)
$$


where $g(t)$ is the motion of the base in the y-direction and $h(t)$ is the small rotation of the base.

Substituting (3) into (2), we have

$$
\begin{aligned}
& \frac{\partial^{2} M(x, t)}{\partial x^{2}}+c_{s} I \frac{\partial^{5} w_{r e l}(\mathrm{x}, \mathrm{t})}{\partial x^{4} \partial t}+c_{a} \frac{\partial w_{r e l}(x, t)}{\partial t} \\
& \quad+m \frac{\partial^{2} w_{r e l}(x, t)}{\partial t^{2}} \\
& =-m \frac{\partial^{2} w_{b}(x, t)}{\partial t^{2}}-c_{a} \frac{\partial w_{b}(x, t)}{\partial t}-c_{s} I \frac{\partial^{5} w_{b}(\mathrm{x}, \mathrm{t})}{\partial x^{4} \partial t} \\
& \quad+f(x, t)
\end{aligned}
$$

Since both the viscous air damping and internal strain damping are related to the relative speed of beam motion, and the tip mass $M_{t}$ is introduced into the above equation, then (4) can be rewritten as

$$
\begin{aligned}
& \frac{\partial^{2} M(x, t)}{\partial x^{2}}+c_{s} I \frac{\partial^{5} w_{r e l}(x, \mathrm{t})}{\partial x^{4} \partial t}+c_{a} \frac{\partial w_{r e l}(x, t)}{\partial t} \\
& \quad+m \frac{\partial^{2} w_{r e l}(x, t)}{\partial t^{2}} \\
& =-\left[m+M_{t} \delta(x-L)\right] \frac{\partial^{2} w_{b}(x, t)}{\partial t^{2}}
\end{aligned}
$$

where $\delta(x)$ is the Dirac delta function. The internal bending moment in (5) can be written as

$$
\begin{aligned}
& M(x, t)=-b\left(\int_{-h_{p}-h_{s} / 2}^{-h_{s} / 2} T^{P} y d y\right. \\
& \left.\quad+\int_{-h_{s} / 2}^{h_{s} / 2} T^{S} y d y+\int_{h_{s} / 2}^{h_{p}+h_{s} / 2} T^{P} y d y\right)
\end{aligned}
$$

where $b$ is the width of the bimorph cantilever beam, $h_{p}$ is the thickness of each piezoceramic layer, and $h_{s}$ is the thickness of the substructure layer, as shown in Figure 1(b). Moreover, $T^{P}$ and $T^{S}$ are the axial stress components in the piezoceramic and substructure layers, respectively, which can be given by

$$
\begin{aligned}
& T^{P}=c_{11}^{E} \delta_{1}^{p}-e_{31} E_{3}, \\
& T^{S}=Y_{S} \delta_{1}^{s}
\end{aligned}
$$

where $c_{11}^{E}$ is the elastic stiffness of the piezoceramic layer at constant electric field, $e_{31}$ is the piezoelectric constant, $E_{3}$ is the electric field component, $Y_{S}$ is Young's modulus of the substructure layer, and $\delta_{1}^{p}$ and $\delta_{1}^{s}$ are the axial strain components in the piezoelectric and substructure layers, respectively. Since the axial strain at a certain position $y$ from the neutral axis of the composite beam is simply proportional to the curvature of the beam at position $x, \delta_{1}^{p}$ and $\delta_{1}^{s}$ can be given by

$$
\delta_{1}^{p}(x, y, t)=\delta_{1}^{s}(x, y, t)=-y \frac{\partial^{2} w_{r e l}(x, t)}{\partial x^{2}}
$$

For the configuration with parallel connection shown in Figure 2(a), the top and the bottom piezoceramic layers are assumed to be identical, and $e_{31}$ has the same sign in top and bottom piezoceramic layers, whereas the instantaneous electric fields are in the opposite directions, i.e., $E_{3}=-v(t) /$ $h_{p}$ in the top layer and $E_{3}=v(t) / h_{p}$ in the bottom layer. Since the piezoelectric coupling term in (6) is only a function of time, before substituting (6) into (5), the electrical term must be multiplied by $[H(x)-H(x-L)]$, where $H(x)$ is the Heaviside function.

With the above discussion, the coupled beam equation can be derived as follows:

$$
\begin{aligned}
Y I & \frac{\partial^{4} w_{r e l}(x, t)}{\partial x^{4}}+c_{s} I \frac{\partial^{5} w_{r e l}(x, t)}{\partial x^{4} \partial t}+c_{a} \frac{\partial w_{r e l}(x, t)}{\partial t} \\
& +m \frac{\partial^{2} w_{r e l}(x, t)}{\partial t^{2}}+\vartheta v(t)\left[\frac{d \delta(x)}{d x}-\frac{d \delta(x-L)}{d x}\right] \\
& =-\left[m+M_{t} \delta(x-L)\right] \frac{\partial^{2} w_{b}(x, t)}{\partial t^{2}}
\end{aligned}
$$

where $\vartheta$ is the backward coupling term, YI is the backward coupling term, and $m$ is the mass per unit length term, which are, respectively, defined as

$$
\begin{aligned}
\vartheta & =\frac{e_{31} b}{h_{p}}\left[\frac{h_{s}^{2}}{4}-\left(h_{p}+\frac{h_{s}}{2}\right)^{2}\right] \\
Y I & =\frac{2 b}{3}\left[Y_{s} \frac{h_{s}^{3}}{8}+c_{11}^{E}\left(\left(h_{p}+\frac{h_{s}}{2}\right)^{3}-\frac{h_{s}^{3}}{8}\right)\right] \\
m & =b\left(\rho_{s} h_{s}+2 \rho_{p} h_{p}\right)
\end{aligned}
$$

where $\rho_{s}$ and $\rho_{p}$ are the mass densities of the substructure and the piezoceramic materials, respectively.

According to the above preliminary, we build the schematic of the V-shaped piezoelectric energy harvester and set the corresponding vibration reference coordinate system, as shown in Figure 3. In this study, we only consider the first two-order vibration modes and ignore the rotary inertias of two beams, when establishing the model of the V-shaped structure by the segment modeling method.

As shown in Figure 3, beam 1 is not subject to the axial load $\left(M_{t 1}\right.$, beam 2 and $M_{t 2}$ can be regarded as an integrated mass), and the absolute displacement of the base in $X_{1}$ $Y_{1}$ coordinate system can be assumed to be zero. Similarly, beam 2 is subject to the axial load $M_{t 2} g \sin \theta$, and the absolute displacement of the base in $X_{2}-Y_{2}$ coordinate system can be assumed to be zero. Hence, by the use of (9), the electromechanical coupling vibration equations of beam 1 and beam 2 can be, respectively, written as

$$
\begin{aligned}
& Y_{1} I_{1} \frac{\partial^{4} w_{r e l 1}\left(x_{1}, t\right)}{\partial x_{1}^{4}}+c_{s 1} I_{1} \frac{\partial^{5} w_{r e l 1}\left(x_{1}, t\right)}{\partial x_{1}^{4} \partial t} \\
&+c_{a 1} \frac{\partial w_{r e l 1}\left(x_{1}, t\right)}{\partial t}+m_{1} \frac{\partial^{2} w_{r e l 1}\left(x_{1}, t\right)}{\partial t^{2}} \\
& \quad+\vartheta_{1} v_{1}(t)\left[\frac{d \delta\left(x_{1}\right)}{d x_{1}}-\frac{d \delta\left(x_{1}-L_{1}\right)}{d x_{1}}\right]=0
\end{aligned}
$$




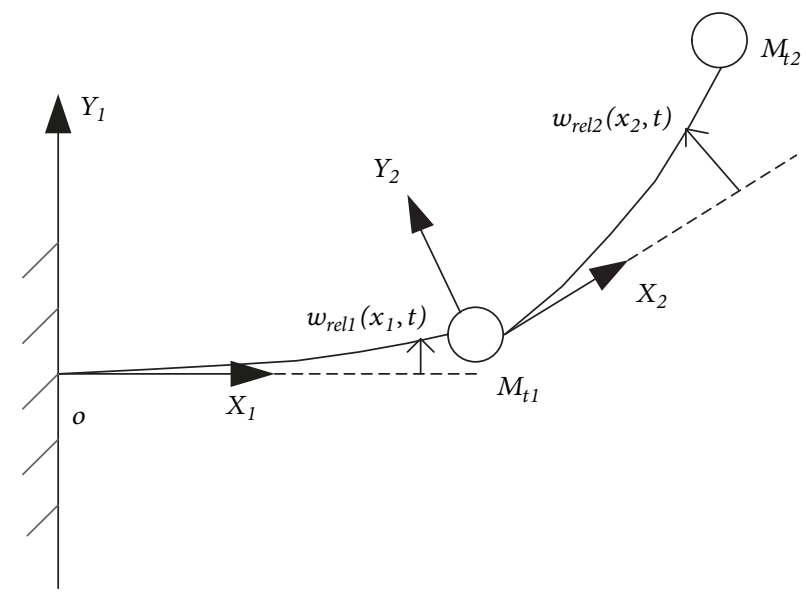

FIGURE 3: The vibration schematic of the V-shaped structure.

$$
\begin{aligned}
Y_{2} & I_{2} \frac{\partial^{4} w_{r e l 2}\left(x_{2}, t\right)}{\partial x_{2}^{4}}+c_{s 1} I_{1} \frac{\partial^{5} w_{r e l 2}\left(x_{2}, t\right)}{\partial x_{2}^{4} \partial t} \\
& +c_{a 1} \frac{\partial w_{r e l 2}\left(x_{2}, t\right)}{\partial t}+m_{2} \frac{\partial^{2} w_{r e l 2}\left(x_{2}, t\right)}{\partial t^{2}} \\
& +M_{t 2} g \sin \theta \frac{\partial^{2} w_{r e l 2}\left(x_{2}, t\right)}{\partial x_{2}^{2}} \\
& +\vartheta_{2} v_{2}(t)\left[\frac{d \delta\left(x_{2}\right)}{d x_{2}}-\frac{d \delta\left(x_{2}-L_{2}\right)}{d x_{2}}\right]=0
\end{aligned}
$$

where $Y_{1} I_{1}$ and $Y_{2} I_{2}$ are the bending stiffness of beam 1 and beam 2, respectively; $m_{1}$ and $m_{2}$ are the mass per unit length of beam 1 and beam 2, respectively; $\vartheta_{1}$ and $\vartheta_{2}$ are the backward coupling coefficient, respectively; $c_{s 1} I_{1}$ and $c_{s 2} I_{2}$ are the internal strain damping of beam 1 and beam 2, respectively; $c_{a 1}$ and $c_{a 2}$ are the viscous air damping of beam 1 and beam 2 , respectively; $v_{1}(t)$ and $v_{2}(t)$ are the output voltage of beam 1 and beam 2, respectively; $\delta(\mathrm{x})$ is the Dirac function; $w_{\text {rel } 1}\left(x_{1}, t\right)$ is the transverse vibratory displacement of beam 1 relative to the substrate; and $w_{\text {rel } 2}\left(x_{2}, t\right)$ is the transverse vibratory displacement of beam 2 relative to the mass.

At the clamped end $\left(x_{1}=0\right)$, both the transverse vibratory displacement and angular displacement equal 0 ; thus the boundary conditions can be written as

$$
\begin{array}{r}
w_{r e l 1}(0, t)=0 \\
\left.\frac{\partial w_{r e l 1}\left(x_{1}, t\right)}{\partial x_{1}}\right|_{x_{1}=0}=0
\end{array}
$$

At $x_{2}=0$ (as well as $x_{1}=L_{1}$ ), the transverse vibratory displacement in local coordinate system equals 0 , and by using the angular displacement equilibrium relations, we have

$$
\begin{aligned}
w_{r e l 2}(0, t) & =0, \\
\left.\frac{\partial w_{r e l 1}\left(x_{1}, t\right)}{\partial x_{1}}\right|_{x_{1}=L_{1}} & =\left.\frac{\partial w_{r e l 2}\left(x_{2}, t\right)}{\partial x_{2}}\right|_{x_{2}=0}
\end{aligned}
$$

At $x_{2}=0$ and $x_{2}=L_{2}$, the force and moment equilibrium relations are, respectively, given by

$$
\begin{aligned}
& Y_{1} I_{1}\left.\frac{\partial^{3} w_{r e l 1}\left(x_{1}, t\right)}{\partial x_{1}^{3}}\right|_{x_{1}=L_{1}} \\
&=\left.M_{t 1} \frac{\partial^{2} w_{r e l 1}\left(x_{1}, t\right)}{\partial t^{2}}\right|_{x_{1}=L_{1}} \\
&+\left.m_{2} L_{2} \cos \theta \frac{\partial^{2} w_{r e l 2}\left(x_{2}, t\right)}{\partial t^{2}}\right|_{x_{2}=L_{2} / 2} \\
&+\left.M_{t 2} \cos \theta \frac{\partial^{2} w_{r e l 2}\left(x_{2}, t\right)}{\partial t^{2}}\right|_{x_{2}=L_{2}} \\
&\left.Y_{1} I_{1} \frac{\partial^{2} w_{r e l 1}\left(x_{1}, t\right)}{\partial x_{1}^{2}}\right|_{x_{1}=L_{1}}+\left.I_{a 1} \frac{\partial^{3} w_{r e l 1}\left(x_{1}, t\right)}{\partial t^{2} \partial x_{1}}\right|_{x_{1}=L_{1}} \\
&=\left.Y_{2} I_{2} \frac{\partial^{2} w_{r e l 2}\left(x_{2}, t\right)}{\partial x_{2}^{2}}\right|_{x_{2}=0}+\frac{m_{2} g L_{2}}{2} \cos \theta \\
& \quad+M_{t 2} g L_{2} \cos \theta \\
&\left.M_{t 2} g \frac{\partial w_{r e l 2}\left(x_{2}, t\right)}{\partial x_{2}}\right|_{x_{2}=L_{2}}+\left.Y_{2} I_{2} \frac{\partial^{3} w_{r e l 2}\left(x_{2}, t\right)}{\partial x_{2}^{3}}\right|_{x_{2}=L_{2}} \\
&=\left.\left.M_{t 2} \frac{\partial^{2} w_{r e l 2}\left(x_{2}, t\right)}{\partial t^{2}}\right|_{x_{2}=L_{2}} \frac{\partial^{2} w_{r e l 2}\left(x_{2}, t\right)}{\partial x_{2}^{2}}\right|_{x_{2}=L_{2}}+\left.I_{a 2} \frac{\partial^{3} w_{r e l 2}\left(x_{2}, t\right)}{\partial t^{2} \partial x_{2}}\right|_{x_{2}=L_{2}} \\
&=0
\end{aligned}
$$

where $I_{a 1}$ and $I_{a 2}$ are the rotary inertias of the tip masses $M_{t 1}$ and $M_{t 2}$, respectively. 


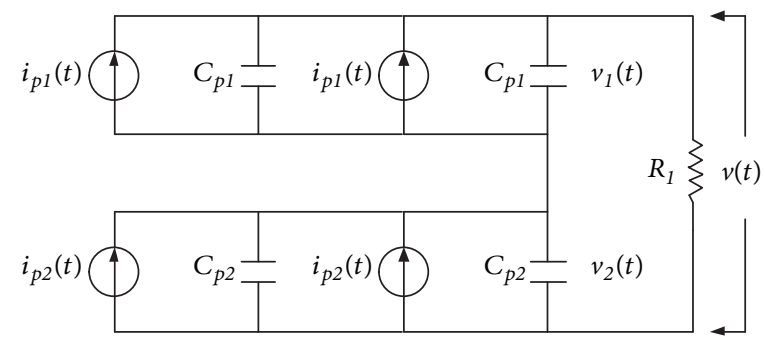

FIGURE 4: Electrical circuit of the condition that the two beams connect in series.

Based on the expansion theorem, the vibratory motion of a beam segment can be represented by an absolutely and uniformly convergent series of the eigenfunctions as

$$
w_{\text {relk }}\left(x_{k}, t\right)=\sum_{r=1}^{\infty} \phi_{k r}\left(x_{k}\right) \eta_{r}(t)
$$

where $\phi_{k r}\left(x_{k}\right)$ is the normalized mass eigenfunction and $\eta_{r}(t)$ is the modal response of the $r$ th vibration mode; $k$ denotes the serial number of the beam. With (13) and (14), by ignoring the internal and external damping and separating the variables, the piecewise-defined eigenfunctions can be written as

$$
\begin{aligned}
\phi_{1 r}\left(x_{1}\right)= & A_{1 r} \sin \left(\alpha_{r} x_{1}\right)+B_{1 r} \cos \left(\alpha_{r} x_{1}\right) \\
& +C_{1 r} \sinh \left(\alpha_{r} x_{1}\right)+D_{1 r} \cosh \left(\alpha_{r} x_{1}\right) \\
\phi_{2 r}\left(x_{2}\right)= & A_{2 r} \sin \left(\beta_{r} x_{2}\right)+B_{2 r} \cos \left(\beta_{r} x_{2}\right) \\
& +C_{2 r} \sinh \left(\gamma_{r} x_{2}\right)+D_{2 r} \cosh \left(\gamma_{r} x_{2}\right)
\end{aligned}
$$

where

$$
\begin{aligned}
& \alpha_{r}^{4}=\omega_{r}^{2} \frac{m_{1}}{Y_{1} I_{1}}, \\
& \beta_{r}^{2}=\frac{\kappa}{2}+\sqrt{\frac{\kappa^{2}}{4}+\omega_{r}^{2} \frac{m_{2}}{Y_{2} I_{2}}} \\
& \gamma_{r}^{2}=-\frac{\kappa}{2}+\sqrt{\frac{\kappa^{2}}{4}+\omega_{r}^{2} \frac{m_{2}}{Y_{2} I_{2}}}, \quad \kappa=\frac{M_{t 2} g}{Y_{2} I_{2}}
\end{aligned}
$$

where $\omega_{r}$ is the undamped natural frequency of $r$ th vibration mode, and it can be calculated by the proposed method in [41].

Substituting (21)-(25) into (15)-(20) results in an $8 \times 8$ coefficient matrix, and we can obtain the value of $A_{1 r} \sim D_{2 r}$ by solving this matrix. After substituting (21) into (13) and (14), and by applying the orthogonality condition of normalized mass eigenfunctions, we can write the mechanical vibratory equation in modal coordinates as

$$
\begin{aligned}
& \frac{d^{2} \eta_{r}(t)}{d t^{2}}+2 \zeta_{r} \omega_{r} \frac{d \eta_{r}(t)}{d t}+\omega_{r}^{2} \eta_{r}(t)+\chi_{1 r} v_{1}(t) \\
& +\chi_{2 r} \nu_{2}(t)=f_{r}(t)
\end{aligned}
$$

where $v_{1}(t)$ and $v_{2}(t)$ are the voltages of the crossbeam and the inclined beam, respectively; $\chi_{k r}$ is the modal electromechanical coupling term; $f_{r}(t)$ is the modal mechanical response function. $\chi_{k r}$ and $f_{r}(t)$ can be calculated by

$$
\begin{aligned}
\chi_{k r} & =\left.\vartheta_{k} \frac{d \phi_{k r}\left(x_{k}\right)}{d x_{k}}\right|_{0} ^{L_{k}} \\
f_{r}(t) & =-m_{1}\left(\frac{d^{2} g(t)}{d t^{2}} \int_{0}^{L_{1}} \phi_{1 r}\left(x_{1}\right) d x_{1}\right. \\
& \left.+\frac{d^{2} h(t)}{d t^{2}} \int_{0}^{L_{1}} x_{1} \phi_{1 r}\left(x_{1}\right) d x_{1}\right)-\left(M_{t 1}+M_{t 2}\right. \\
& \left.+m_{2} L_{2}\right) \phi_{1 r}\left(L_{1}\right)\left(\frac{d^{2} g(t)}{d t^{2}}+L_{1} \frac{d^{2} h(t)}{d t^{2}}\right)
\end{aligned}
$$

2.3. Coupled Circuit Equations. In this study, we only consider the condition that the crossbeam connects with the inclined beam in series, and each piezoelectric layer can be seen as the current source connecting with the capacitor in parallel, as shown in Figure 4. The current sources produced by beam 1 and beam 2 can be written as

$$
i_{p k}(t)=\sum_{r=1}^{\infty} \psi_{k r} \frac{d \eta_{r}(t)}{d t}
$$

where

$$
\psi_{k r}=-\left.\left(e_{31}\right)_{k}\left(h_{p c}\right)_{k} b_{k} \frac{d \phi_{k r}\left(x_{k}\right)}{d x_{k}}\right|_{0} ^{L_{k}}
$$

In (30), $\left(e_{31}\right)_{k}$ is the piezoelectric constant, $\left(h_{p c}\right)_{k}$ is the distance between the center of the piezoceramic layer and the neutral axis of the cross-section, and $b_{k}$ is the width of piezoceramic layer. The internal capacitor $C_{p k}$ can be written as

$$
C_{p k}=\frac{\left(\varepsilon_{33}^{S}\right)_{k} b_{k} L_{k}}{\left(h_{p}\right)_{k}}
$$

where $\left(\varepsilon_{33}^{S}\right)_{k}$ is the permittivity of the piezoceramic layer at constant strain, $L_{k}$ is the length of beam, and $\left(h_{p}\right)_{k}$ is the thickness of the piezoceramic layer. 
TABLE 1: The material and dimension parameters of piezoelectric bimorph and substructure.

\begin{tabular}{lc}
\hline Material and geometric parameters & Value $/$ Unit \\
\hline PZT-51 Density, $\rho_{p}$ & $7.45 \times 10^{3} \mathrm{~kg} / \mathrm{m}^{3}$ \\
PZT-51 Elastic stiffness constant, $c_{11}{ }^{2}$ & $76.9 \mathrm{GPa}$ \\
PZT-51 Piezoelectric constant, $d_{31}$ & $-186 \times 10^{-12} \mathrm{C} / \mathrm{N}$ \\
PZT-51 Permittivity, $\varepsilon_{33}{ }^{S}$ & $1500 \varepsilon_{0} \mathrm{~F} / \mathrm{m}$ \\
Density of copper substructure, $\rho_{s}$ & $9.0 \times 10^{3} \mathrm{~kg} / \mathrm{m}^{3}$ \\
Young's modulus of copper substructure, $Y$ & $106 \mathrm{GPa}$ \\
PZT-51 Geometry $\left(L \times b \times h_{p}\right)$ & $70 \mathrm{~mm} \times 15 \mathrm{~mm} \times 0.55 \mathrm{~mm}$ \\
Geometry of copper substructure $\left(L \times b \times h_{s}\right)$ & $70 \mathrm{~mm} \times 18 \mathrm{~mm} \times 0.45 \mathrm{~mm}$ \\
\hline
\end{tabular}

Subsequently, as the bimorph piezoelectric plates connect in series, with the Kirchhoff Laws, we can write the coupled circuit equations as

$$
\begin{aligned}
& C_{p 1} \frac{d v_{1}(t)}{d t}+\frac{v_{1}(t)}{2 R_{1}}+\frac{v_{2}(t)}{2 R_{1}}=\sum_{r=1}^{\infty} \psi_{1 r} \frac{d \eta_{r}(t)}{d t} \\
& C_{p 2} \frac{d v_{2}(t)}{d t}+\frac{v_{2}(t)}{2 R_{1}}+\frac{v_{1}(t)}{2 R_{1}}=\sum_{r=1}^{\infty} \psi_{2 r} \frac{d \eta_{r}(t)}{d t}
\end{aligned}
$$

By solving (26) and (32), $v_{1}(t), v_{2}(t)$, and $\eta_{r}(t)$ can be obtained. With $v_{1}(t)$ and $v_{2}(t)$, we can calculate the voltage across the load resistance $R_{1}$ by

$$
v(t)=v_{1}(t)+v_{2}(t)
$$

2.4. Coupled Voltage Response for Harmonic Base Excitation. Assuming that the electromechanical system is linear, the modal mechanical response function can be written as $f_{r}(t)=F_{r} e^{j \omega t}$. Besides, the steady state expressions for the modal response and voltage response of two beams can be represented by harmonic functions: $\eta_{r}(t)=H_{r} e^{j \omega t}, v_{1}(t)=$ $V_{1} e^{j \omega t}$, and $v_{2}(t)=V_{2} e^{j \omega t}$. Substituting the above equations into (26) and (32), the two equations can be, respectively, written as

$$
\begin{aligned}
& \left(\omega_{r}^{2}-\omega^{2}+j 2 \zeta_{r} \omega_{r} \omega\right) H_{r}+\chi_{1 r} V_{1}+\chi_{2 r} V_{2}=F_{r} \\
& \left(\frac{1}{2 R_{1}}+j \omega C_{p 1}\right) V_{1}+\frac{V_{2}}{2 R_{1}}-j \omega \sum_{r=1}^{\infty} \psi_{1 r} H_{r}=0 \\
& \left(\frac{1}{2 R_{1}}+j \omega C_{p 2}\right) V_{2}+\frac{V_{1}}{2 R_{1}}-j \omega \sum_{r=1}^{\infty} \psi_{2 r} H_{r}=0
\end{aligned}
$$

Substituting (34) into (33), we have

$$
\begin{aligned}
& Q_{11} V_{1}+Q_{12} V_{2}=S_{1} \\
& Q_{21} V_{1}+Q_{22} V_{2}=S_{2}
\end{aligned}
$$

where

$$
\begin{aligned}
Q_{m k} & =\frac{1}{2 R_{1}}+j \omega C_{p m} \delta_{m k}+\sum_{r=1}^{\infty} \frac{j \omega \psi_{m r} \chi_{k r}}{\omega_{r}^{2}-\omega^{2}+j 2 \zeta_{r} \omega_{r} \omega} \\
S_{m} & =\sum_{r=1}^{\infty} \frac{j \omega \psi_{m r} F_{r}}{\omega_{r}^{2}-\omega^{2}+j 2 \zeta_{r} \omega_{r} \omega}
\end{aligned}
$$

From (36), two maximum voltage amplitudes $\left(V_{1}\right.$ and $\left.V_{2}\right)$ of the closed-loop circuit are obtained. Substituting $V_{1}$ and $V_{2}$ into $v_{1}(t)=V_{1} e^{j \omega t}$ and $v_{2}(t)=V_{2} e^{j \omega t}$, respectively, we can obtain the steady state voltage response functions produced by the two beams. Then, the output voltage $v(t)$ of the Vshaped bimorph piezoelectric cantilever is given by

$$
v(t)=v_{1}(t)+v_{2}(t)
$$

\section{Numerical Simulation}

In this section, the voltage performance of the V-shaped cantilevered piezoelectric bimorph harvester is simulated by Matlab. Lead zirconate titanate (PZT) is a ceramic perovskite material that has a prominent piezoelectric effect; thus this material has been widely used in various engineering applications. Since the $d_{51}$ mode can potentially improve the energy harvesting performance of piezoelectric energy harvester [42], and the PZT-51 is commercially available, we use PZT51 material in the proposed energy harvesting structure. The cross beam and inclined beam have the same geometric and material parameters, which are listed in Table 1. In order to achieve resonant excitation, we can adjust their harmonic frequency by the use of tip masses of cross beam and inclined beam $\left(M_{t 1}\right.$ and $\left.M_{t 2}\right)$. In this study, $M_{t 1}$ and $M_{t 2}$ are set as $14 \mathrm{~g}$ and $14.6 \mathrm{~g}$, respectively.

Assuming that beam 1 and beam 2 intersect at an angle of $90^{\circ}$, the values of load resistance are, respectively, set as $1 \mathrm{~K} \Omega, 33 \mathrm{~K} \Omega, 100 \mathrm{~K} \Omega$, and $220 \mathrm{~K} \Omega$. When the two beams connect in series, the voltage frequency response functions (FRFs) for four different load resistances are, respectively, shown in Figure 5. From this figure, we can see that there are two close peak voltages in the output of $\mathrm{V}$-shaped harvester, and the two peak frequencies, respectively, correspond to the first natural frequency and second natural frequency of this harvester, while the single cantilevered piezoelectric beam only has one natural frequency. It immediately follows that the frequency band is successfully broadened. It can be also seen from Figure 5 that the output peak voltage gradually increases with the increase of load resistance value, and the first natural frequency and second natural frequency increase slightly with the increase of load resistance value. Since the first natural frequency and second natural frequency are close to each other, the proposed energy harvester can effectively increase the operation bandwidth and is more applicable 

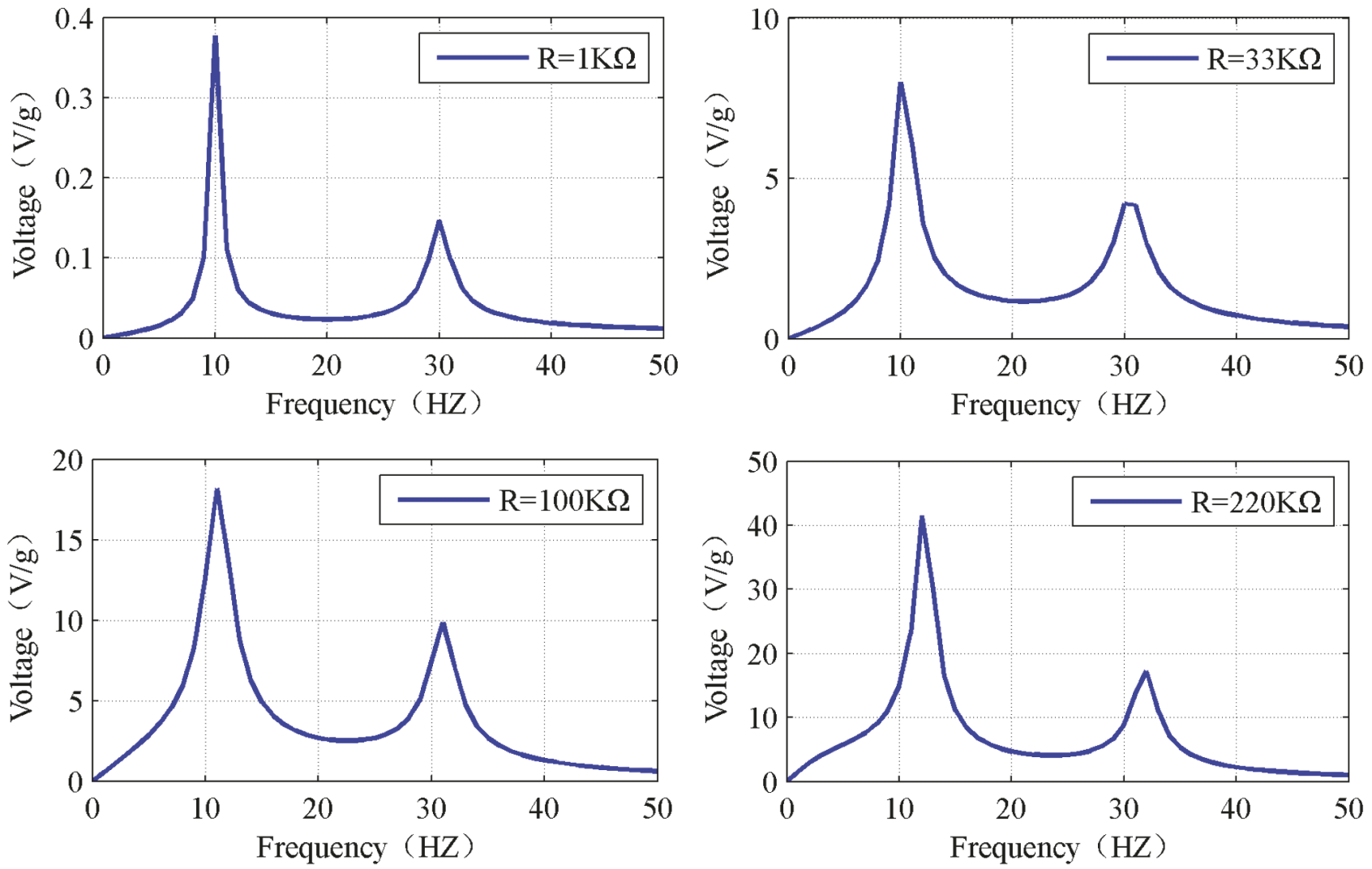

FIGURE 5: Voltage FRFs for four different load resistances.

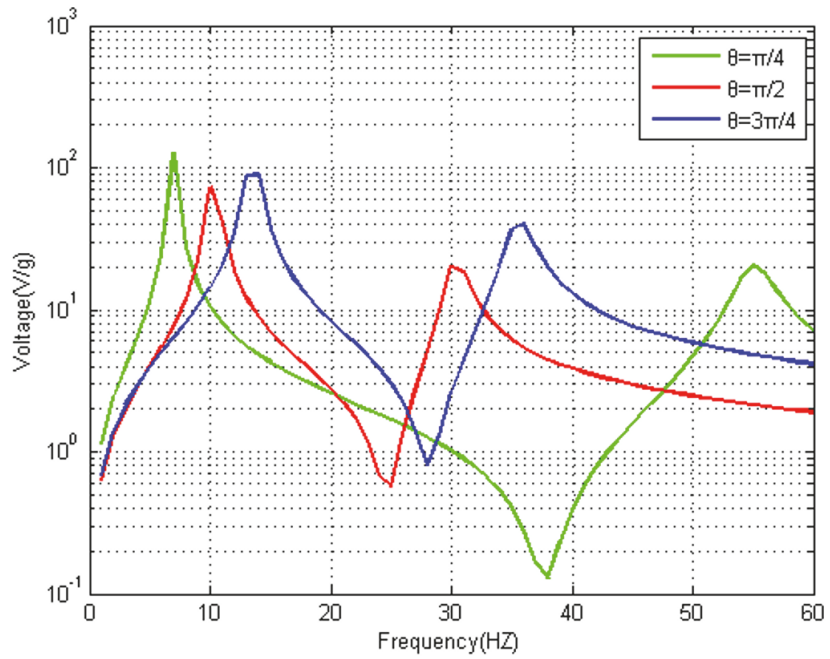

FIgURE 6: Voltage FRFs at different $\theta$.

to the actual environment compared to the conventional cantilevered harvester.

Obviously, the value of $\theta$ may also affect the natural frequency of this system. Assuming that $\theta$ equals to $\pi / 4, \pi / 2$, and $3 \pi / 4$, respectively, and the external load resistance is $100 \mathrm{~K} \Omega$, then the output voltage frequency responses with different $\theta$ are, respectively, illustrated in Figure 6. It can be seen from Figure 6 that when $\theta$ equals to $\pi / 4$, the difference between the first natural frequency and the second natural frequency is the largest; thus the effect of bandwidth extension is not satisfactory in such case. Moreover, when $\theta$ equals $\pi / 2$ or $3 \pi / 4$, their voltage output performance are similar, whereas the difference between the first natural frequency and the second natural frequency by calculation is the smallest when $\theta$ equals $3 \pi / 4$.

$\mathrm{Su}$ et al. [11] proposed a unimorph $\mathrm{V}$-shaped piezoelectric harvester. The first beam is a cantilevered beam with tip mass while the second beam is attached to the end of the first beam 
TABLE 2: Comparison of the newly bimorph V-shaped harvester with the previous unimorph harvester in [11] (load resistance=50 $\mathrm{k} \Omega$ ).

\begin{tabular}{|c|c|c|c|c|}
\hline \multicolumn{2}{|c|}{$\theta$} & $\pi / 4$ & $\pi / 2$ & $3 \pi / 4$ \\
\hline \multirow{2}{*}{ Vlotage (V/g) } & Present device & 9.67 & 8.78 & 8.93 \\
\hline & $\mathrm{Su}[11]$ & 3.01 & 2.96 & 2.47 \\
\hline \multirow{2}{*}{ Power $\left(\mathrm{mW} / \mathrm{g}^{2}\right)$} & Present device & 1.87 & 1.54 & 1.59 \\
\hline & $\mathrm{Su}[11]$ & 0.18 & 0.17 & 0.12 \\
\hline
\end{tabular}

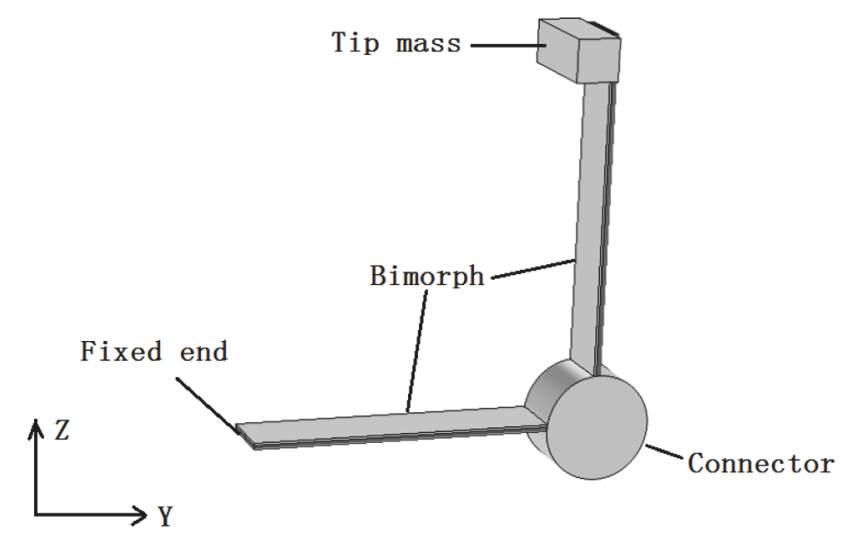

FIGURE 7: The schematic of the proposed vibration energy harvester.

with a certain angle. Piezoelectric layers are attached to both beams in unimorph configuration for power generation. Su investigated the influence of $\theta$ on the frequency responses and plotted the curves of the frequency response of voltage and power under different $\theta$. The peak voltage and power are important indicators which can reflect the performance of an energy harvester. For comparison, the simulations for both the unimorph piezoelectric harvester and the bimorph V-shaped piezoelectric harvester are performed based on the same material and geometric parameters used in [11], and the corresponding peak voltages and powers at the first resonant frequency are obtained. The comparative results are listed in Table 2.

It can be seen from Table 2 that both the peak voltage and power at the first resonant frequency generated by the previous unimorph V-shaped harvester in [11] are lower than those generated by the proposed bimorph V-shaped harvester in this study under different $\theta$. While the two kinds of energy harvester have the similar structure, the bimorph configuration has a better effect in improving the output performance than the unimorph configuration. In other words, with the same material and geometric parameters, the newly bimorph V-shaped harvester is able to generate more power than the previous unimorph V-shaped harvester and thus is more practical in vibration energy harvesting.

\section{Experiment}

4.1. Structure of Energy Harvester. The V-shaped vibration energy harvester consists of two piezoelectric bimorphs, and its schematic is shown in Figure 7.

The used piezoelectric bimorph is produced by the PANT, as shown in Figure 8(a), and its key parameters have been
TABLE 3: The masses of all the components in the proposed harvester.

\begin{tabular}{lc}
\hline Component & Mass $(\mathrm{g})$ \\
\hline Bimorph & 12 \\
Connector & 14 \\
Tip mass & 14.6 \\
\hline
\end{tabular}

listed in Table 1. The two cantilevered piezoelectric beams constitute the proposed harvester via a connector, which is shown in Figure 8(b). This cylindrical connector is made of aluminum alloy, and it has eight narrow slots which are manufactured by linear cutting in the axial direction. Thus, we can connect the two piezoelectric cantilevers at one given angle. This connector can be also regarded as a tip mass. The tip mass is made of wolfram steel with a high mass density, which is beneficial to tune the natural frequency. The masses of all components in the designed harvester are listed in Table 3.

4.2. Energy Harvester Test System. The experimental flow chart and setup of the V-shaped energy harvester are, respectively, illustrated in Figures 9 and 10. In the process of testing, an actuator (BK4809) which is driven by a power amplifier (BK2706) and a signal generator (Agilent 33521A) is used to generate sinusoidal excitations with different amplitudes and frequencies. Then the output voltage and current under the excitation can be measured by the multimeter (VC9808). The acceleration sensor (LC0103T) is used to measure the vibration acceleration, whose waveform is displayed by the digital oscilloscope 54645D. And the amplitude of the excitation signal is tuned by the power amplifier. 


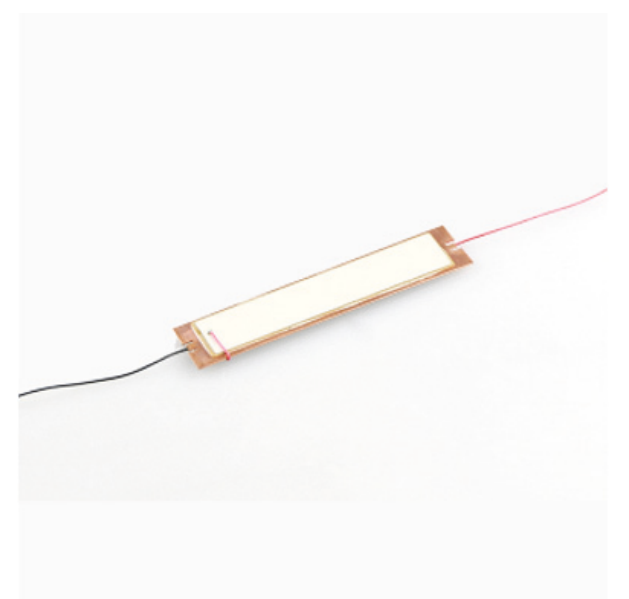

(a)

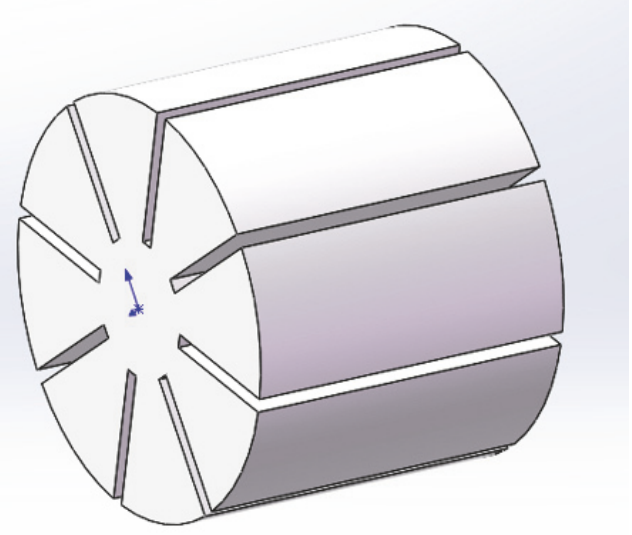

(b)

FIGURE 8: The key components of the proposed harvester: (a) piezoelectric bimorph and (b) connector.

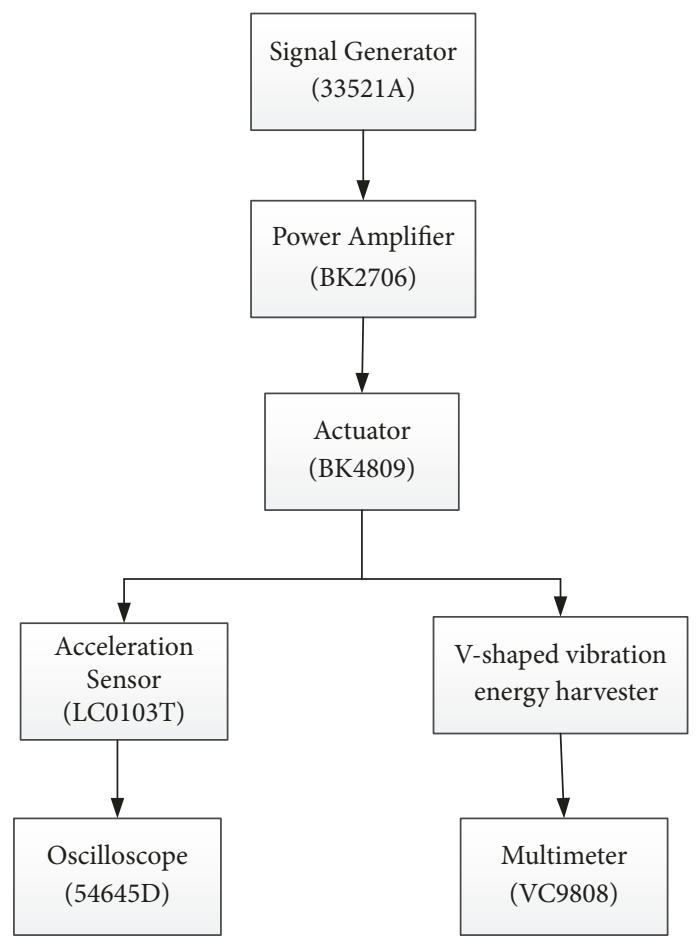

FIGURE 9: The flow chart of the performance measurement for the proposed harvester.

4.3. Experimental Results. Firstly, we test the output performance of this $\mathrm{V}$-shaped energy harvester under certain excitation in order to validate the proposed mathematic model. Let the angle of $\theta$ equal $45^{\circ}, 90^{\circ}$, and $135^{\circ}$, respectively, and the corresponding experimental setups of $\mathrm{V}$-shaped energy harvester are, respectively, shown in Figures 11(a)-11(c).

The open circuit voltage versus frequency curves at different $\theta$ are shown in Figure 12. It is easy to note from Figure 12 that whenever the angle of $\theta$ is $45^{\circ}$ or $90^{\circ}$ or $135^{\circ}$, there are two peak values of open circuit voltage at the first natural frequency and the second natural frequency. The frequency response characteristic of the frequency range between the two natural frequencies (i.e., the operation frequency band) can be used to evaluate the performance of energy harvesting. Obviously, when $\theta$ is $45^{\circ}$, the operation frequency band is the widest, whereas its frequency response characteristic is not satisfactory; when $\theta$ is $135^{\circ}$, compared to the L-shaped structure (i.e., $\theta$ is $90^{\circ}$ ), not only is the operation frequency band wider (see Table 4), but also its frequency response characteristic is better. Furthermore, when $\theta$ is $45^{\circ}$ and $135^{\circ}$, both the obtained open circuit voltages under the first natural frequency excitation are much larger than that obtained by the L-shaped structure. Therefore, it can be concluded that the $\mathrm{V}$-shaped structure has a better voltage 


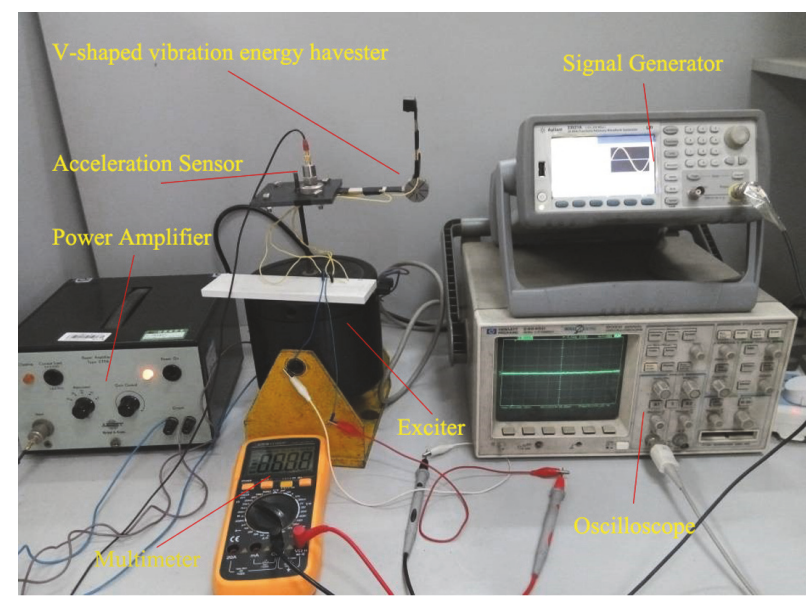

FIGURE 10: Measurement setup for the proposed harvester.

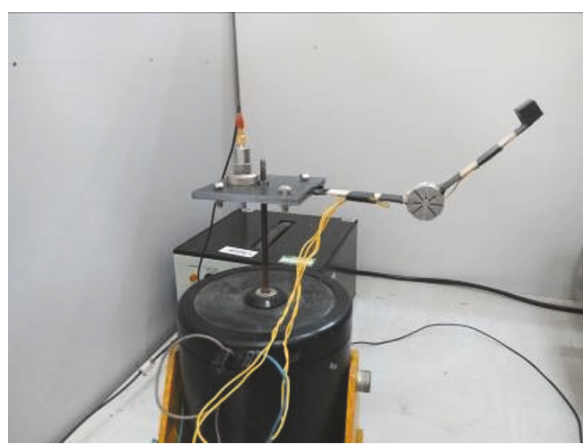

(a)

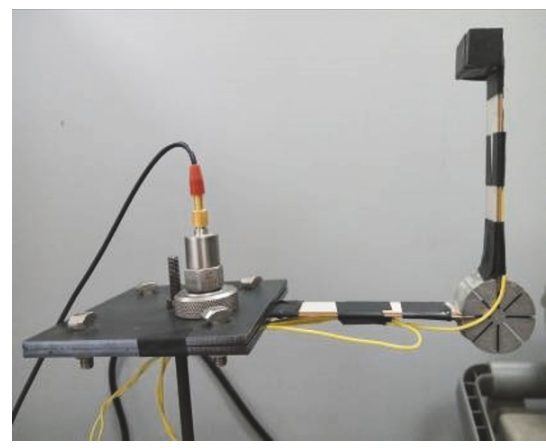

(b)

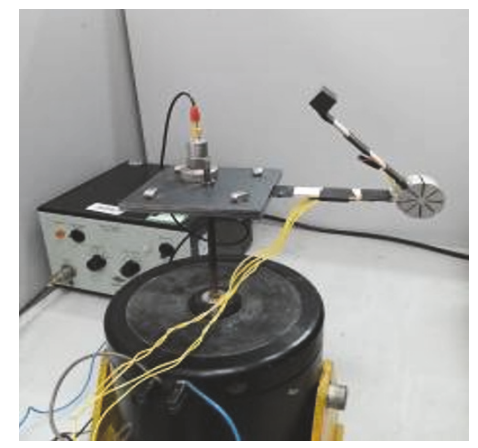

(c)

Figure 11: The experimental setups under the $z$-axis excitation: (a) $\theta=45^{\circ}$, (b) $\theta=90^{\circ}$, and (c) $\theta=135^{\circ}$.

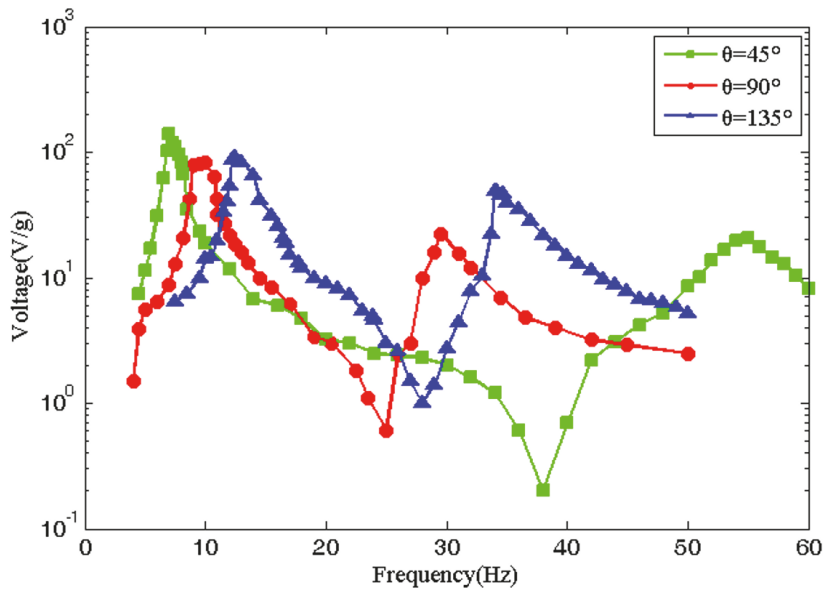

FIGURE 12: The measured open circuit voltage versus exciting frequency curves at different $\theta$.

output performance and wider operation bandwidth than the L-shaped structure in these cases.

The simulation and experimental results of V-shaped energy harvester under different conditions are compared, which is shown in Figure 13, and the natural frequency and the corresponding open circuit voltage are summarized in Table 4. It can be known from Figure 13 and Table 4 that the experimental results are close to the simulation results, which verifies the proposed mathematical model. It should be noted that the experimental results are a little higher than the simulation results. According to the mechanism of the piezoelectricity, there are three modes, $d_{31}, d_{32}, d_{33}$, which would transform the deformation into energy. In general, the $d_{32}$ and $d_{33}$ mode hardly do contribution to the 
TABLE 4: The simulation and experimental results under different conditions.

\begin{tabular}{|c|c|c|c|c|c|}
\hline$\theta$ & Types & $\begin{array}{l}\text { The first natural } \\
\text { frequency }(\mathrm{Hz})\end{array}$ & $\begin{array}{l}\text { The second } \\
\text { natural } \\
\text { frequency }(\mathrm{Hz})\end{array}$ & $\begin{array}{c}\text { Open circuit voltage } \\
\text { under the first natural } \\
\text { frequency excitation } \\
(\mathrm{V} / \mathrm{g})\end{array}$ & $\begin{array}{c}\text { Open circuit voltage } \\
\text { under the second natural } \\
\text { frequency excitation } \\
(\mathrm{V} / \mathrm{g})\end{array}$ \\
\hline \multirow{2}{*}{$\theta=45^{\circ}$} & Experimental value & 7 & 54 & 139 & 21.6 \\
\hline & Simulation value & 7 & 55 & 127.00 & 20.48 \\
\hline \multirow{2}{*}{$\theta=90^{\circ}$} & Experimental value & 9.2 & 29 & 83.2 & 22.9 \\
\hline & Simulation value & 10 & 30 & 72.87 & 20.18 \\
\hline \multirow{2}{*}{$\theta=135^{\circ}$} & Experimental value & 12.5 & 34.2 & 91.6 & 49.1 \\
\hline & Simulation value & 14 & 36 & 88.70 & 40.32 \\
\hline
\end{tabular}

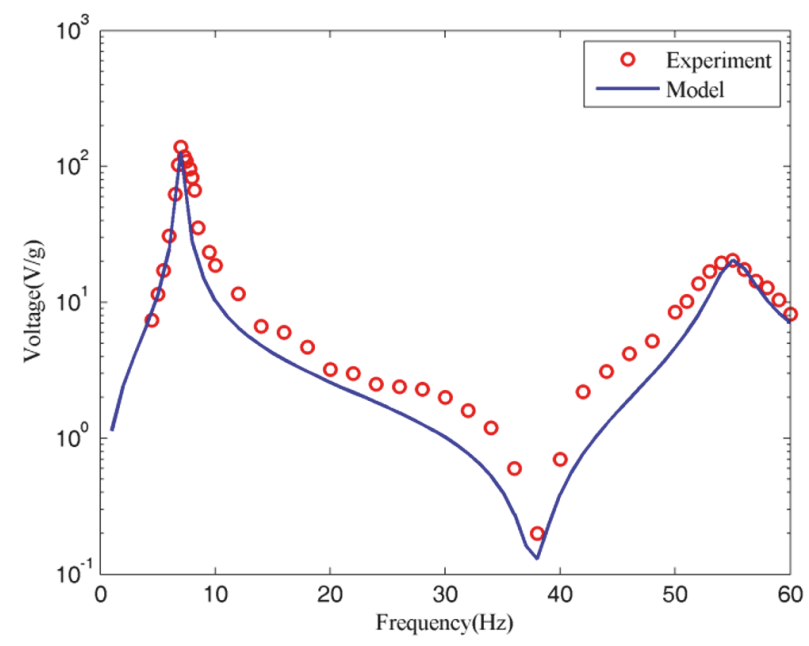

(a)

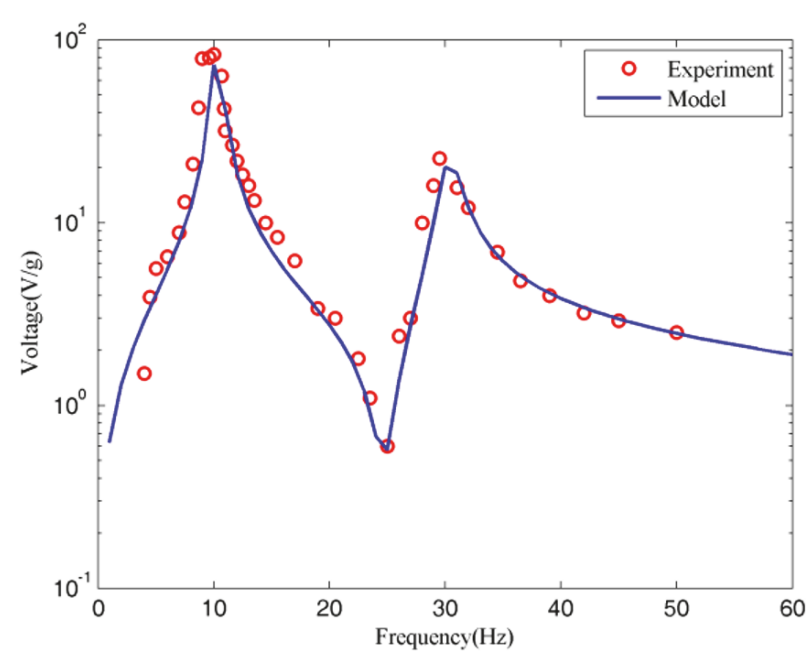

(b)

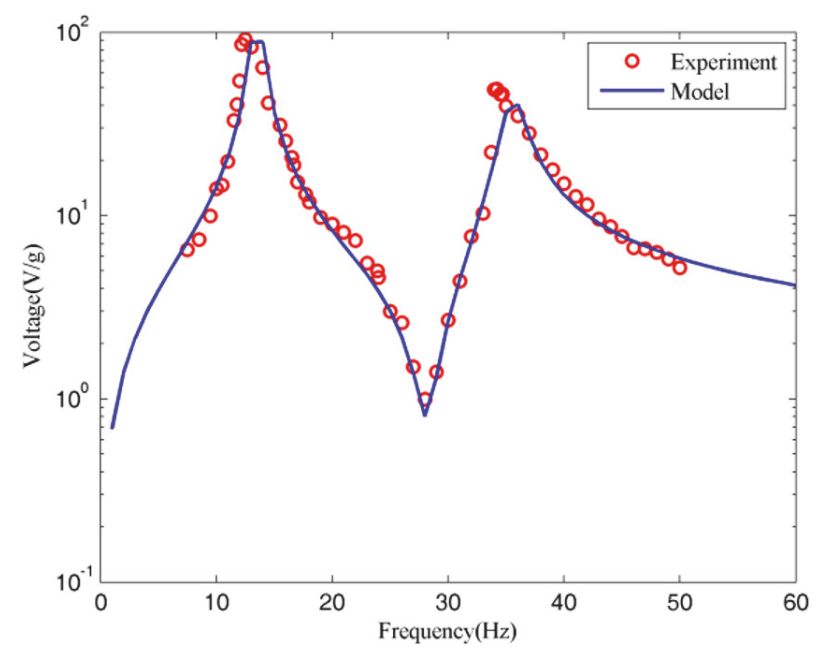

(c)

FIGURE 13: The comparison between the experimental measurements and model predictions at (b) $\theta=45^{\circ},(\mathrm{c}) \theta=90^{\circ}$, and (d) $\theta=135^{\circ}$.

energy generation, so in this paper we only consider the $\mathrm{d}_{31}$ mode; the transverse deformation of the cantilever beam can generate the maximum energy. In order to simplify the model, the $\mathrm{d}_{32}$ and $\mathrm{d}_{33}$ mode are ignored. But the cantilever beams did deform in vertical direction during experiment, so the experimental results are a little higher than the simulation results under some excitations. In addition, there might be some experimental errors. The amplifier is tuned manually, and the amplitude of the actual excitation may be higher than that of the theoretical excitation, which leads to the fact that the experimental values are higher than the theoretical ones.

To adapt various ambient excitations, we can conveniently tune the angle of $\mathrm{V}$-shaped energy harvester by the connector, which leads to the change of the operation 


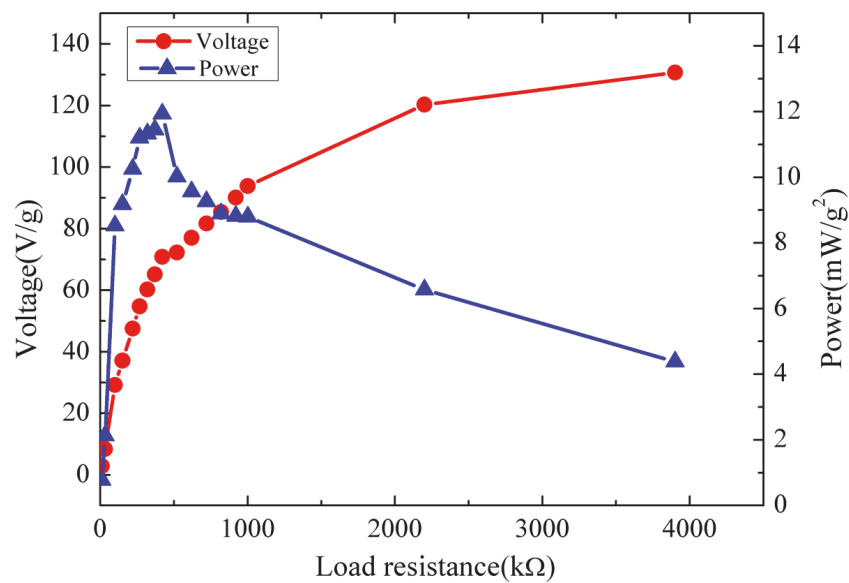

(a)

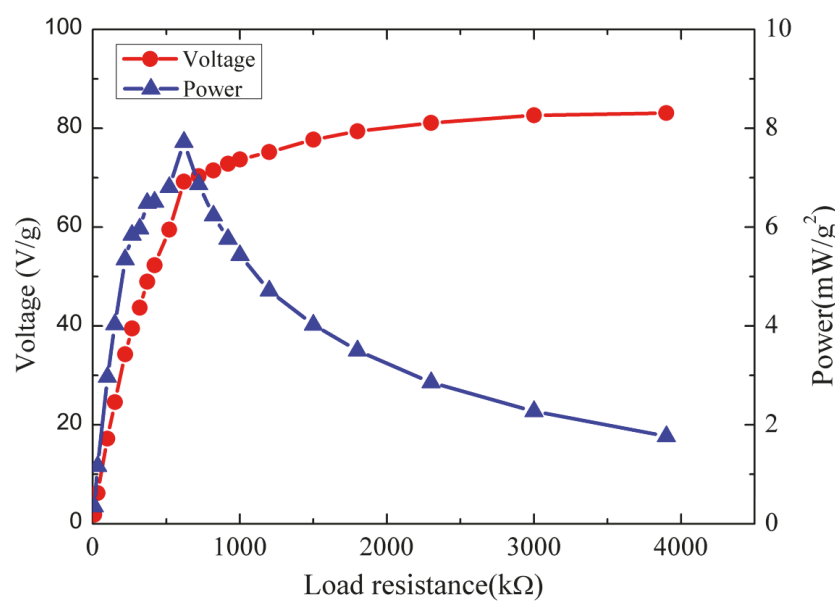

(c)

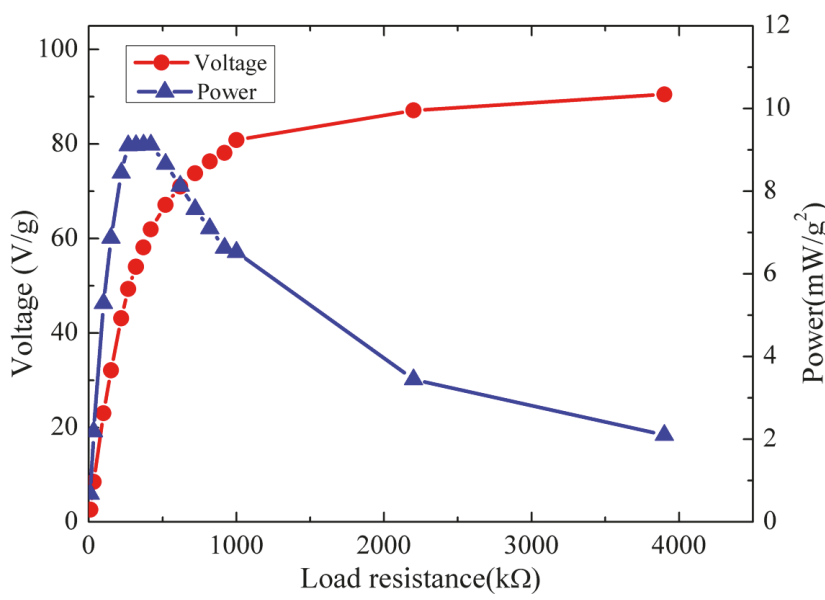

(e)

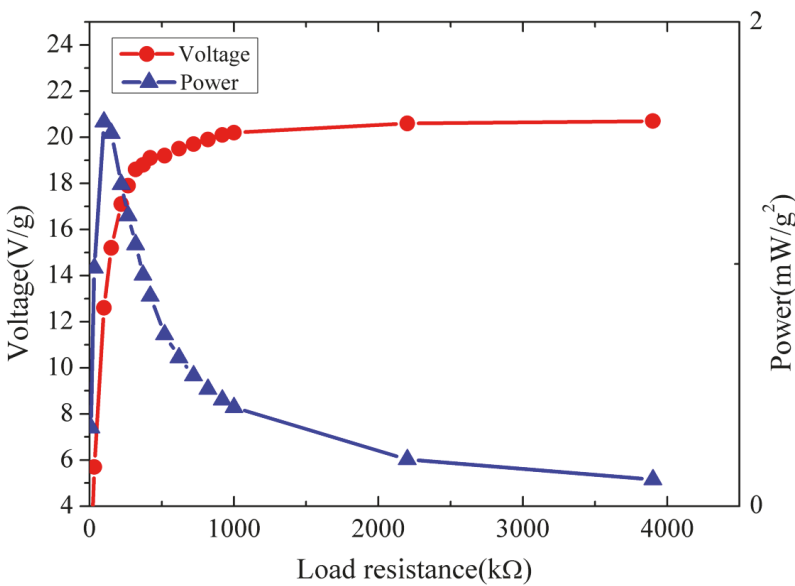

(b)

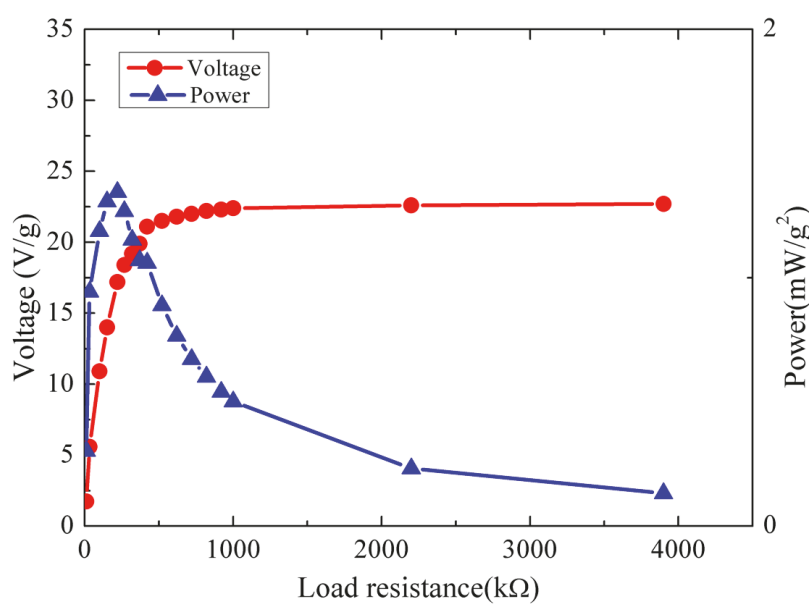

(d)

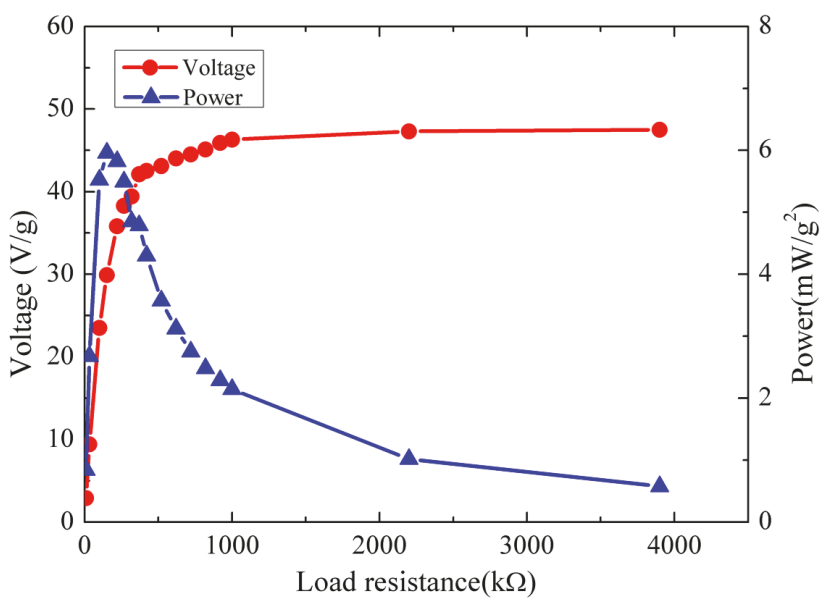

(f)

FIGURE 14: The output voltage and output power versus load resistance curves under $z$-axis excitation: (a) $\theta=45^{\circ}$, the first natural frequency excitation; (b) $\theta=45^{\circ}$, the second natural frequency excitation; (c) $\theta=90^{\circ}$, the first natural frequency excitation; (d) $\theta=90^{\circ}$, the second natural frequency excitation; (e) $\theta=135^{\circ}$, the first natural frequency excitation; (f) $\theta=135^{\circ}$, the second natural frequency excitation. 
bandwidth. However, for other commonly used harvesters, tuning the mass may be a conventional and effective method. Obviously, the tuning method based on the proposed harvester is more convenient.

Then we consider the impedance matching of the proposed energy harvester under different structure parameters and ambient excitations. When the three kinds of $\mathrm{V}$-shaped energy harvesters are excited at the first natural frequency and the second natural frequency under different load resistance, the measured output voltage and power versus load resistance curves are simultaneously plotted in Figure 14. It can be seen from this figure that the output voltage will increase with the increase of the load resistance, and the output voltage will be close to the open circuit voltage when the resistance is up to a certain value. However, for the output power, it will firstly increase and then decrease with the increase of load resistance. There is a maximum output power, and the corresponding load resistance is the best matched resistance.

\section{Conclusions}

Since the ambient vibration is usually broadband and changeable, a novel $\mathrm{V}$-shaped vibration energy harvester is proposed based on the cantilevered piezoelectric bimorph structure. This harvester consists of two cantilevered piezoelectric bimorph beams which intersect at a certain angle. By using the Euler-Bernoulli equation and piezoelectric equation, the mechanical coupling equations of the V-shaped energy harvester are established. And then the coupled circuit equation is derived by Kirchhoff's laws. With the above equations, the output responses of the $\mathrm{V}$-shaped harvester under different structural parameters and load resistances are calculated, and it can be seen from the simulation results that there is a wide operation band between the first natural frequency and the second natural frequency. At last, various experiments are carried out to demonstrate the output performance of the proposed energy harvester, and the obtained results show that (1) the natural frequency and operation bandwidth of the V-shaped structure can be easily tuned by the use of the connector to adapt the ambient vibration; (2) the operation frequency band of $\mathrm{V}$-shaped structure and its corresponding frequency response characteristic can be improved for some $\theta$; (3) the V-shaped structure has a better voltage output performance than the L-shaped structure and the V-shaped unimorph structure in the given examples; (4) the maximum output power can be achieved by resistance matching under the excitation of natural frequency. Therefore, the proposed $\mathrm{V}$-shaped energy harvester can not only increase the output of the electrical energy from the environmental vibration, but also conveniently tune the operation bandwidth, which make it more applicable in various environments. In further study, an adaptive connector (such as motor and spring) can be designed to make the $\mathrm{V}$-shaped energy harvester self-tune with the change of ambient excitation.

\section{Conflicts of Interest}

The authors declare that they have no conflicts of interest.

\section{Acknowledgments}

The work described in this paper is supported by National Natural Science Foundation of China (no. 51675065), Chongqing Research Program of Basic Research and Frontier Technology (no. cstc2017jcyjAX0459), the project for industrial transformation and foundation enhancement funded in 2014, and State Key Laboratory of Mechanical Transmission funded project (no. SKLMT-ZZKT-2015Z14).

\section{References}

[1] C. Cheng, Z. Chen, H. Shi, Z. Liu, and Y. Xiong, "System-Level Coupled Modeling of Piezoelectric Vibration Energy Harvesting Systems by Joint Finite Element and Circuit Analysis," Shock and Vibration, vol. 2016, Article ID 2413578, 2016.

[2] I. Korhonen and R. Lankinen, "Energy harvester for a wireless sensor in a boiler environment," Measurement, vol. 58, pp. 241248, 2014.

[3] J. Zhang, W. Li, N. Han, and J. Kan, "Forest fire detection system based on a ZigBee wireless sensor network," Frontiers of Forestry in China, vol. 3, no. 3, pp. 369-374, 2008.

[4] S. Ullah, H. Higgins, B. Braem et al., "A comprehensive survey of wireless body area networks on PHY, MAC, and network layers solutions," Journal of Medical Systems, vol. 36, no. 3, pp. 10651094, 2012.

[5] C. Li, R.-V. Sanchez, G. Zurita, M. Cerrada, D. Cabrera, and R. E. Vásquez, "Multimodal deep support vector classification with homologous features and its application to gearbox fault diagnosis," Neurocomputing, vol. 168, pp. 119-127, 2015.

[6] Y. Luo, H. Sun, X. Wang, L. Zuo, and N. Chen, "Wind Induced Vibration Control and Energy Harvesting of Electromagnetic Resonant Shunt Tuned Mass-Damper-Inerter for Building Structures," Shock and Vibration, vol. 2017, Article ID 4180134, 2017.

[7] S. Roundy, P. K. Wright, and J. M. Rabaey, Energy Scavenging for Wireless Sensor Networks, Springer, New York, NY, USA, 2004.

[8] J. G. Rocha, L. M. Gonçalves, P. F. Rocha, M. P. Silva, and S. Lanceros-Méndez, "Energy harvesting from piezoelectric materials fully integrated in footwear," IEEE Transactions on Industrial Electronics, vol. 57, no. 3, pp. 813-819, 2010.

[9] J. Lee and B. Choi, "Development of a piezoelectric energy harvesting system for implementing wireless sensors on the tires," Energy Conversion and Management, vol. 78, pp. 32-38, 2014.

[10] P. Gambier, S. R. Anton, N. Kong, A. Erturk, and D. J. Inman, "Piezoelectric, solar and thermal energy harvesting for hybrid low-power generator systems with thin-film batteries," Measurement Science and Technology, vol. 23, no. 1, Article ID 015101, 2012.

[11] W. Su and J. W. Zu, "Modeling of V-Shaped Beam-Mass Piezoelectric Energy Harvester: Impact of the Angle Between the Beams," in Proceedings of the ASME 2012 International Mechanical Engineering Congress and Exposition, p. 573, Houston, Texas, USA.

[12] C. Williams and R. B. Yates, "Analysis Of A Micro-electric Generator For Microsystems," in Proceedings of the International Solid-State Sensors and Actuators Conference - TRANSDUCERS '95, pp. 369-372, Stockholm, Sweden. 
[13] S. L. Kok, N. M. White, and N. R. Harris, "Free-standing thickfilm piezoelectric multimorph cantilevers for energy harvesting," in Proceedings of the 2009 IEEE International Ultrasonics Symposium, IUS 2009, ita, September 2009.

[14] A. Erturk and D. J. Inman, "An experimentally validated bimorph cantilever model for piezoelectric energy harvesting from base excitations," Smart Materials and Structures, vol. 18, no. 2, Article ID 025009, 2009.

[15] R. S. Langley, "A general mass law for broadband energy harvesting," Journal of Sound and Vibration, vol. 333, no. 3, pp. 927-936, 2014.

[16] N. G. Elvin and A. A. Elvin, "A general equivalent circuit model for piezoelectric generators," Journal of Intelligent Material Systems and Structures, vol. 20, no. 1, pp. 3-9, 2009.

[17] J. M. Renno, M. F. Daqaq, and D. J. Inman, "On the optimal energy harvesting from a vibration source using a piezoelectric stack," Energy Harvesting Technologies, pp. 165-194, 2009.

[18] H. Li, C. Tian, and Z. D. Deng, "Energy harvesting from low frequency applications using piezoelectric materials," Applied Physics Reviews, vol. 1, no. 4, p. 041301, 2014.

[19] A. R. M. Siddique, S. Mahmud, and B. V. Heyst, "A comprehensive review on vibration based micro power generators using electromagnetic and piezoelectric transducer mechanisms," Energy Conversion and Management, vol. 106, pp. 728-747, 2015.

[20] R. L. Harne and K. W. Wang, "A review of the recent research on vibration energy harvesting via bistable systems," Smart Materials and Structures, vol. 22, no. 2, Article ID 023001, 2013.

[21] S. Leadenham and A. Erturk, "M-shaped asymmetric nonlinear oscillator for broadband vibration energy harvesting: Harmonic balance analysis and experimental validation," Journal of Sound and Vibration, vol. 333, no. 23, pp. 6209-6223, 2014.

[22] R. L. Harne, A. Sun, and K. W. Wang, "Leveraging nonlinear saturation-based phenomena in an L-shaped vibration energy harvesting system," Journal of Sound and Vibration, vol. 363, pp. 517-531, 2016

[23] F. Danzi, J. M. Gibert, G. Frulla, and E. Cestino, "Graph-based element removal method for topology synthesis of beam based ground structures," Structural and Multidisciplinary Optimization, pp. 1-5, 2017.

[24] F. Danzi, J. Gibert, E. Cestino, and G. Frulla, "Topology synthesis of planar ground structures for energy harvesting applications," in Proceedings of the Active and Passive Smart Structures and Integrated Systems 2017, usa, March 2017.

[25] I.-H. Kim, H.-J. Jung, B. M. Lee, and S.-J. Jang, "Broadband energy-harvesting using a two degree-of-freedom vibrating body," Applied Physics Letters, vol. 98, no. 21, Article ID 214102, 2011.

[26] B. Andò, S. Baglio, F. Maiorca, and C. Trigona, "Analysis of two dimensional, wide-band, bistable vibration energy harvester," Sensors and Actuators A: Physical, vol. 202, pp. 176-182, 2013.

[27] W. Su and J. Zu, "An innovative tri-directional broadband piezoelectric energy harvester," Applied Physics Letters, vol. 103, no. 20, p. 203901, 2013.

[28] S. Qi, R. Shuttleworth, S. O. Oyadiji, and J. Wright, "Design of a multiresonant beam for broadband piezoelectric energy harvesting," Smart Materials and Structures, vol. 19, no. 9, Article ID 094009, 2010.

[29] H. Xue, Y. Hu, and Q.-M. Wang, "Broadband piezoelectric energy harvesting devices using multiple bimorphs with different operating frequencies," IEEE Transactions on Ultrasonics, Ferroelectrics and Frequency Control, vol. 55, no. 9, pp. 21042108, 2008.
[30] Z. Yang and J. Yang, "Connected vibrating piezoelectric bimorph beams as a wide-band piezoelectric power harvester," Journal of Intelligent Material Systems and Structures, vol. 20, no. 5, pp. 569-574, 2009.

[31] Y. Hu, H. Xue, and H. Hu, "A piezoelectric power harvester with adjustable frequency through axial preloads," Smart Materials and Structures, vol. 16, no. 5, pp. 1961-1966, 2007.

[32] V. R. Challa, M. G. Prasad, and F. T. Fisher, "Towards an autonomous self-tuning vibration energy harvesting device for wireless sensor network applications," Smart Materials and Structures, vol. 20, no. 2, Article ID 025004, 2011.

[33] M. S. M. Soliman, E. M. Abdel-Rahman, E. F. El-Saadany, and R. R. Mansour, "A design procedure for wideband micropower generators," Journal of Microelectromechanical Systems, vol. 18, no. 6, Article ID 5299263, pp. 1288-1299, 2009.

[34] A. F. Arrieta, T. Delpero, A. E. Bergamini, and P. Ermanni, "Broadband vibration energy harvesting based on cantilevered piezoelectric bi-stable composites," Applied Physics Letters, vol. 102, no. 17, Article ID 173904, 2013.

[35] M. Ferrari, V. Ferrari, M. Guizzetti, B. Andò, S. Baglio, and C. Trigona, "Improved energy harvesting from wideband vibrations by nonlinear piezoelectric converters," Sensors and Actuators A: Physical, vol. 162, no. 2, pp. 425-431, 2010.

[36] S. C. Stanton, C. C. McGehee, and B. P. Mann, "Reversible hysteresis for broadband magnetopiezoelastic energy harvesting," Applied Physics Letters, vol. 95, no. 17, Article ID 174103, 2009.

[37] H. Liu, C. Lee, T. Kobayashi, C. J. Tay, and C. Quan, "Investigation of a MEMS piezoelectric energy harvester system with a frequency-widened-bandwidth mechanism introduced by mechanical stoppers," Smart Materials and Structures, vol. 21, no. 3, Article ID 035005, 2012.

[38] A. Erturk, J. M. Renno, and D. J. Inman, "Modeling of piezoelectric energy harvesting from an L-shaped beam-mass structure with an application to UAVs," Journal of Intelligent Material Systems and Structures, vol. 20, no. 5, pp. 529-544, 2009.

[39] L. Guo and Y. Qin, "Modeling and analysis of a bimorph piezoelectric cantilever," in Proceedings of the 13th International Conference on Ubiquitous Robots and Ambient Intelligence, URAI 2016, pp. 248-251, chn, August 2016.

[40] A. Erturk and D. J. Inman, "On mechanical modeling of cantilevered piezoelectric vibration energy harvesters," Journal of Intelligent Material Systems and Structures, vol. 19, no. 11, pp. 1311-1325, 2008.

[41] A. Erturk and D. J. Inman, "Mechanical considerations for modeling of vibration-based energy harvesters," in Proceedings of the ASME 2007 International Design Engineering Technical Conferences and Computers and Information in Engineering Conference, pp. 769-778, Las Vegas, Nevada, USA, 2007.

[42] J. Zhao, X. Zheng, L. Zhou et al., "Investigation of a d 15 mode PZT-51 piezoelectric energy harvester with a series connection structure," Smart Materials and Structures, vol. 21, no. 10, Article ID 105006, 2012. 


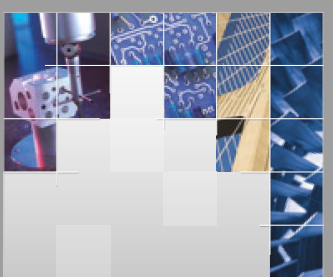

\section{Enfincering}
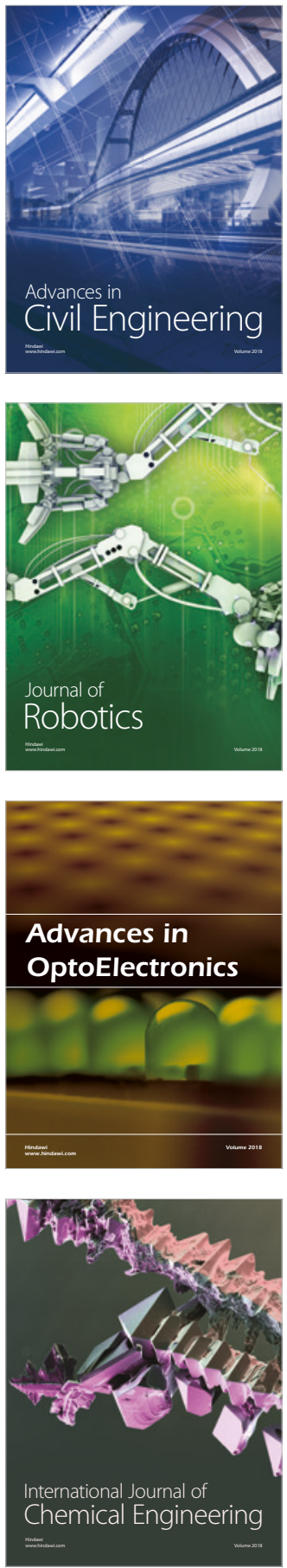

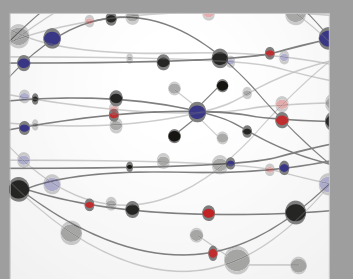

\section{Rotating \\ Machinery}

The Scientific World Journal

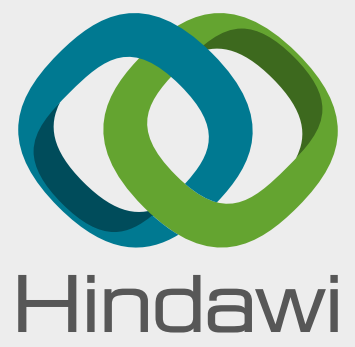

Submit your manuscripts at

www.hindawi.com
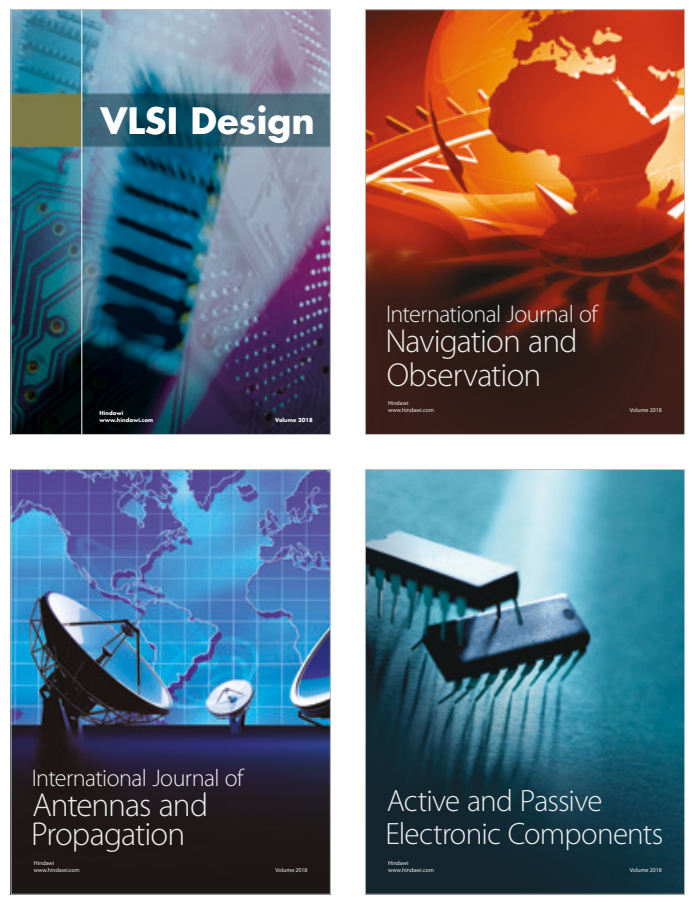
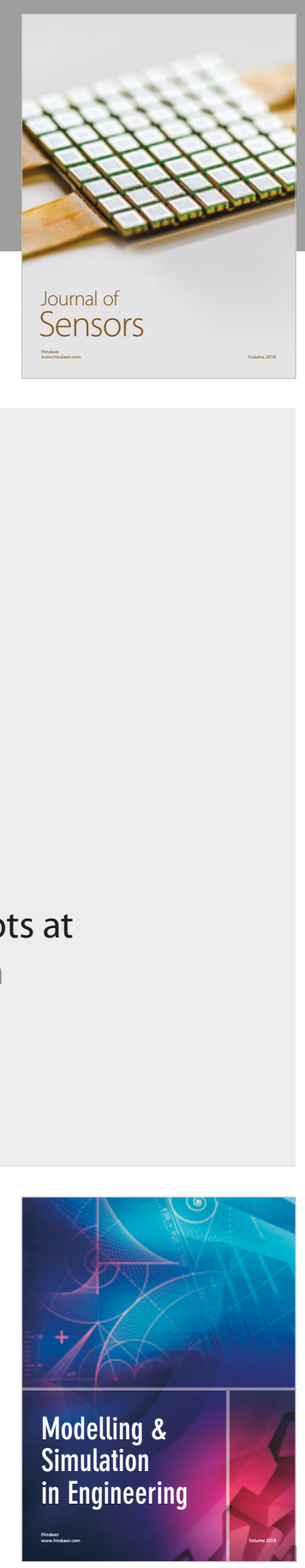

\section{Advances \\ Multimedia}
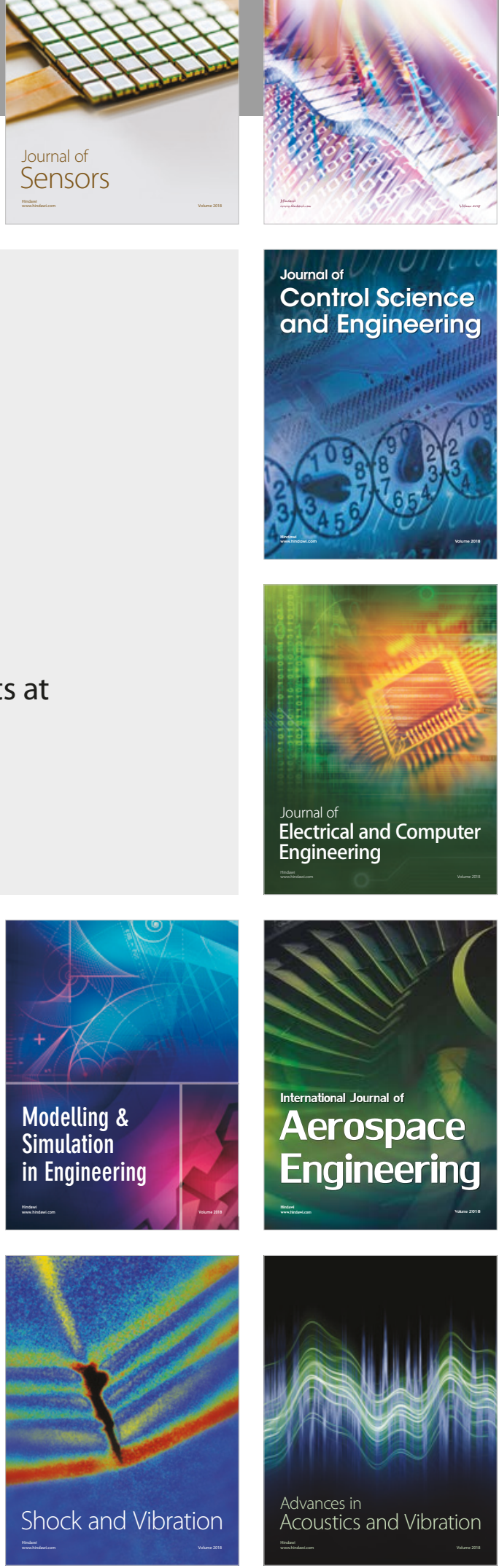